Provided for non-commercial research and education use. Not for reproduction, distribution or commercial use.

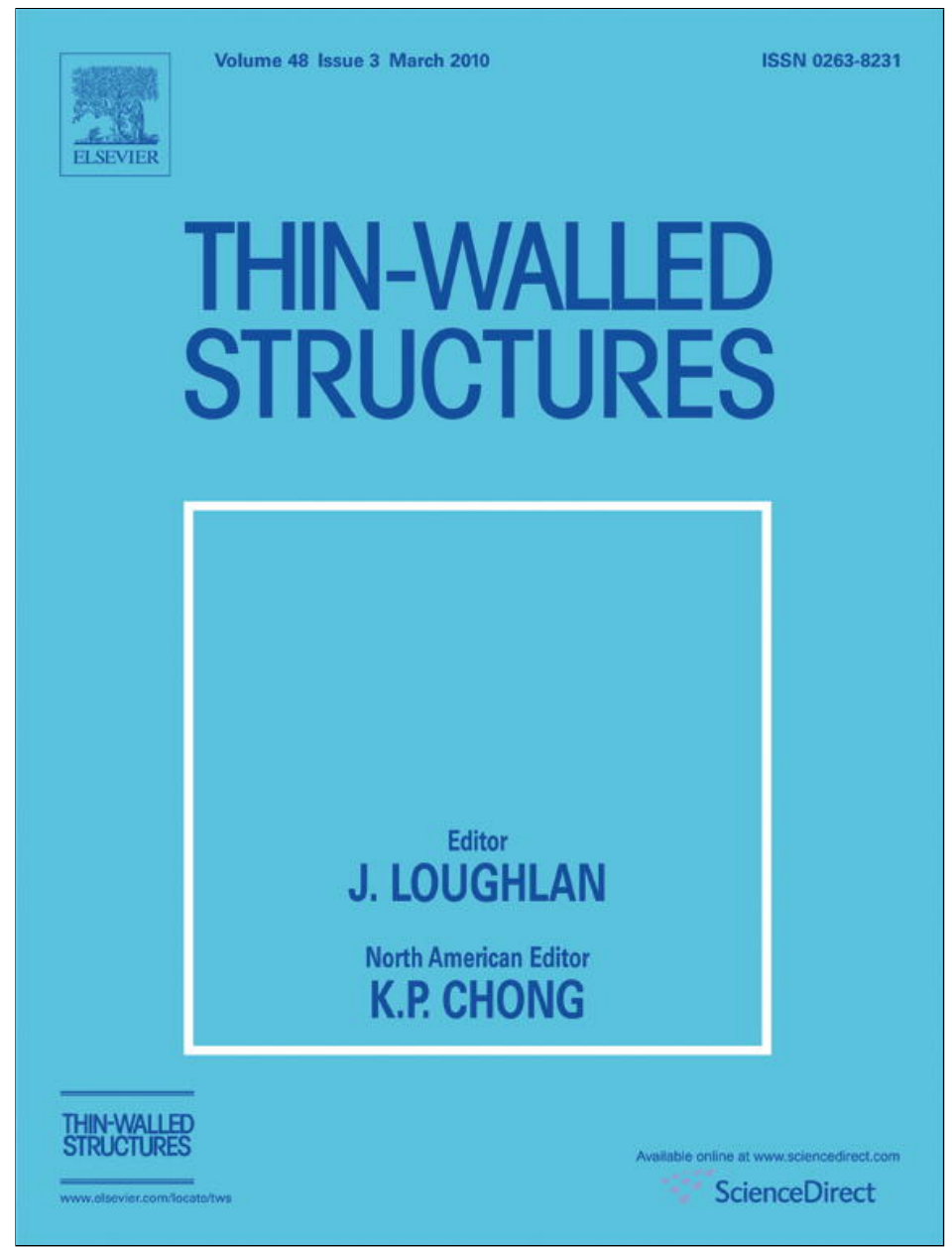

This article appeared in a journal published by Elsevier. The attached copy is furnished to the author for internal non-commercial research and education use, including for instruction at the authors institution and sharing with colleagues.

Other uses, including reproduction and distribution, or selling or licensing copies, or posting to personal, institutional or third party websites are prohibited.

In most cases authors are permitted to post their version of the article (e.g. in Word or Tex form) to their personal website or institutional repository. Authors requiring further information regarding Elsevier's archiving and manuscript policies are encouraged to visit:

http://www.elsevier.com/copyright 


\title{
Empirical formulations for estimation of ultimate strength of continuous stiffened aluminium plates under combined in-plane compression and lateral pressure
}

\author{
Mohammad Reza Khedmati ${ }^{a, *}$, Mohammad Reza Zareei ${ }^{b}$, Philippe Rigo ${ }^{c}$ \\ a Faculty of Marine Technology, Amirkabir University of Technology, Tehran 15914, Iran \\ b Shipbuilding Group, Faculty of Engineering, Chabahar Maritime University, Chabahar 99717-56499, Iran \\ ' University of Liège, ANAST, Chemin des Chevreuils 1 (B52/3), 4000 Liège, Belgium
}

\section{A R T I C L E I N F O}

\section{Article history:}

Received 20 June 2009

Received in revised form

30 September 2009

Accepted 2 October 2009

Available online 7 November 2009

Keywords:

Ultimate strength

Continuous stiffened aluminium plates

Axial compression

Lateral pressure

Empirical formulation

Heat-affected zone

Finite element method (FEM)

Regression analysis

\begin{abstract}
A B S T R A C T
The present research was undertaken based on the results obtained by the same authors in a sensitivity study on the buckling and ultimate strength of continuous stiffened aluminium plates. Empirical expressions are developed for predicting ultimate compressive strength of welded stiffened aluminium plates used in marine applications under combined in-plane axial compression and different levels of lateral pressure. Existing data of the ultimate compressive strength for stiffened aluminium plates numerically obtained by the authors through the previously performed sensitivity analysis are used for deriving formulations that are expressed as functions of two parameters, namely the plate slenderness ratio and the column (stiffener) slenderness ratio. Regression analysis is used in order to derive the empirical formulations. The formulae implicitly include effects of the weld on initial imperfections, and the heat-affected zone.
\end{abstract}

(c) 2009 Elsevier Ltd. All rights reserved.

\section{Introduction}

Stiffened plates are basic building elements in many civil as well as marine structural applications and, as such, accurate strength assessment of individual stiffened plate components is one of the key parameters to perform general strength analysis. These structural components typically consist of a plate with equally spaced stiffeners (bulb, flat bar or T- and L-sections welded on one side) and often with intermediate transverse stiffeners, frames or bulkheads.

Stiffened plates in high-strength aluminium alloys have been used in a variety of marine structures, with applications such as hull and decks in high-speed boats and catamarans and superstructure for ships. Other applications are bridge box girders, and walls and floors of offshore modules and containers. These elements are primarily required to resist axial compressive forces (induced by hull bending moment) as well as lateral loads arising from different sources like hydrostatic/hydrodynamic pressures or cargo weight. Also, other loads such as transverse tension/

\footnotetext{
* Corresponding author. Tel.: +98 2164543113 ; fax: +98 2166412495.

E-mail address: khedmati@aut.ac.ir (M.R. Khedmati).
}

compression, longitudinal tension, in-plane bending moments and shearing forces may act on these structural elements.

The ultimate strength design formulae available for steel plates cannot be directly applied to aluminium plates even if the corresponding material properties are properly accounted for. This is partly due to the fact that the constitutive stress-strain relationship of aluminium alloys is different from that of structural steel. In the elastic-plastic range after the proportional limit as compared with structural steel, strain hardening has a significant influence in the ultimate load behaviour of aluminium structures whereas in steel structures, the elastic-perfectly plastic material model is well adopted. Besides, softening in the heataffected zone (HAZ) significantly affects the ultimate strength behaviour of aluminium structures, whereas its effect in steel structures is of very little importance.

The ultimate strength of stiffened steel plate panels has been the subject of many investigations, both experimentally [1-5] and numerically [6-10], with the most significant contributions in the field of ship structures and bridges. The literature on stiffened aluminium panels is more limited. Clarke and Narayanan [11] report on buckling tests on an aluminium AA5083 plate with welded T-bar and flat-bar stiffeners. His experimental programme comprised eight compression tests on panels with different plate and stiffener sizes, with buckling over two spans as the failure 


\begin{tabular}{|c|c|c|c|}
\hline \multicolumn{2}{|l|}{ Notation } & E & Young's modulus \\
\hline A & cross-sectional area of stiffener with attached plating & & stiffener \\
\hline$A_{P}$ & cross-sectional area of plate & $\sigma$ & average stress \\
\hline$A_{S}$ & cross-sectional area of stiffener & $\sigma_{e l}$ & elastic buckling strength of stiffened plate \\
\hline$L=a$ & length of local plate panels & $\sigma_{c \text {-plate }}$ & elastic buckling strength of stiffened plate for plate \\
\hline$b$ & breadth of local plate panels & & buckling mode \\
\hline$b_{e}$ & $\begin{array}{l}\text { effective breadth of local plate panels } \\
\text { coefficient to define the maximum magnitude of }\end{array}$ & $\sigma_{c \text {-lateral }}$ & $\begin{array}{l}\text { elastic buckling strength of stiffened plate for stiffener } \\
\text { tripping buckling mode }\end{array}$ \\
\hline$C_{1}-C_{5}$ & $\begin{array}{l}\text { initial deflection } \\
\text { constant coefficients }\end{array}$ & $\sigma_{c \text {-torsional }}$ & $\begin{array}{l}\text { elastic buckling strength of stiffened plate for tor- } \\
\text { sional buckling mode }\end{array}$ \\
\hline I & $\begin{array}{l}\text { moment of inertia of a stiffener with its attached } \\
\text { plating }\end{array}$ & $\sigma_{c \text {-web }}$ & $\begin{array}{l}\text { elastic buckling strength of stiffened plate for stiffener } \\
\text { web buckling mode }\end{array}$ \\
\hline $\begin{array}{l}r=(\sqrt{I / A}) \\
t\left(=t_{p}\right)\end{array}$ & $\begin{array}{l}\text { gyration radius of a stiffener with its attached plating } \\
\text { plate thickness }\end{array}$ & $\sigma_{c-\text { flange }}$ & $\begin{array}{l}\text { elastic buckling strength of stiffened plate for stiffener } \\
\text { flange buckling mode }\end{array}$ \\
\hline$t_{w}$ & web thickness of longitudinal stiffener & $\sigma_{Y}$ & yield stress \\
\hline$h_{w}$ & web height of longitudinal stiffener & $\sigma_{Y s}$ & yield stress for the stiffener \\
\hline$t_{f}$ & flange thickness of longitudinal stiffener & $\sigma_{Y p}$ & yield stress of the plate \\
\hline$b_{f}$ & flange breadth of longitudinal stiffener & $\sigma_{Y s e q}$ & equivalent yield stress for the stiffened plate \\
\hline$h$ & water head (pressure) in meters & $\sigma_{U}\left(=\sigma_{u l t}\right)$ & ultimate strength \\
\hline$e_{i}$ & residuals & $\varepsilon$ & average strain \\
\hline$P_{\text {ult }}$ & ultimate load & $\varepsilon_{Y}$ & yield strain \\
\hline$R^{2}$ & parameter for accuracy control of regression model & $\beta$ & slenderness of the plate \\
\hline$x$ and $x_{i}$ & independent variables & $\beta_{j}$ & exact coefficients in the equation of regression models \\
\hline $\begin{array}{l}x_{\max } \text { and } x_{\min } \\
y \text { and } y_{i}\end{array}$ & $\begin{array}{l}\text { in maximum and minimum values, respectively, of } x \\
\text { functional variables }\end{array}$ & $\widehat{\beta}_{j}$ & $\begin{array}{l}\text { estimated coefficients in the equation of regression } \\
\text { models }\end{array}$ \\
\hline$z_{0}$ & $\begin{array}{l}\text { distance between outer surface of plate and neutral } \\
\text { axis of plate-stiffener combination }\end{array}$ & $\lambda$ & $\begin{array}{l}\text { column slenderness of the beam column element } \\
\text { (stiffener and the associated plate) }\end{array}$ \\
\hline$v$ & Poisson's ratio & & \\
\hline
\end{tabular}

mode. The ultimate strength of stiffened aluminium AA6082-T6 plates under axial compression was investigated by Aalberg et al. $[12,13]$ using numerical and experimental methods. Kristensen and Moan [14] demonstrated numerically the effect of HAZ and residual stresses on the ultimate strength of rectangular aluminium plates (AA5083 and AA6082) under bi-axial loading of plates. Some initial experimental and numerical simulations on torsional buckling of flat bars in aluminium panels have been also presented by Zha et al. [15,16] and Zha and Moan [17]. Hopperstad et al. [18] carried out a study with the objective of assessing the reliability of non-linear finite element analyses in predictions on ultimate strength of aluminium plates subjected to in-plane compression. Rigo et al. [19] made a numerical investigation to present reliable finite element models to study the behaviour of axially compressed stiffened aluminium panels (including extruded profiles).

Among most recent works, reference can be made to the work of Paik et al. [20] on the subject of ultimate limit state design of multi-hull ships made in aluminium. The impact of initial imperfections due to the fusion welding on ultimate strength of stiffened aluminium plates was studied by Paik et al. [21] and Collette [22]. Paik et al. [21] defined fabrication related initial imperfections of fusion welded stiffened aluminium plate structures at three levels. Also Paik [23] derived empirical formulations for predicting the ultimate strength of stiffened aluminium plates under axial compression. Future trends and research needs in aluminium structures were outlined by Sielski [24]. Mechanical collapse tests on stiffened aluminium structures for marine applications were performed by Paik et al. [25,26]. Recently, Paik [27] studied buckling collapse testing of friction stir welded aluminium stiffened plate structures. Most recently, Khedmati et al. [28] made an extensive sensitivity analysis on buckling and ultimate strength of continuous stiffened aluminium plates under combined in-plane compression and different levels of lateral pressure.

Following the study made by Khedmati et al. [28] on postbuckling behaviour and ultimate strength characteristics of stiffened aluminium plates under combined axial compressive and lateral pressure loads, a set of empirical formulations or equations are derived in this paper to estimate the ultimate strength of such stiffened plates under above-mentioned load combinations. The ultimate compressive strength data numerically obtained by the authors through their sensitivity analysis [28] are used for deriving the formulations, which are expressed as functions of two parameters, namely the plate slenderness ratio and the column (stiffener) slenderness ratio. Regression analysis is used in order to derive the empirical formulations. The formulae implicitly include effects of weld induced initial imperfections and softening in the heat-affected zone.

\section{Numerical data used for formulae derivations}

\subsection{Structural arrangements and geometrical characteristics of analysed stiffened aluminium plates}

In order to derive the ultimate compressive strength formulae for stiffened aluminium plates, a database of the ultimate strength values for a number of such structural elements is required. The database may be gathered either based on expensive experimental tests or on results of a set of numerical analyses. Thus, in this paper a series of elastic-plastic large deflection analyses is performed applying the finite element method. A total of 199 prototype stiffened aluminium plates were designed for this 
purpose. Geometrical characteristics of the analysed stiffened plates are given in the first 11 columns of Tables 1-6.

As was reported by Paik [23], ultimate compressive strength characteristics of aluminium plates with flat-bar stiffeners differ from those with angle- or T-bar stiffeners. Accordingly, analyses in this paper are done on two different groups of stiffened aluminium plates: a group with flat-bar stiffeners and another one with T-bar stiffeners. Geometrical characteristics of stiffened aluminium plates are chosen so that a wide range of possible plate-stiffener combinations is produced. Fig. 1 shows the crosssectional parameters describing the geometrical characteristics of stiffened plate models.

\subsection{Finite element code and adopted elements}

The ultimate strength of stiffened aluminium plates is hereby assessed using ANSYS [29], in which both material and geometric nonlinearities are taken into account. Among the libraries of available elements of the ANSYS FEM program, the eight-node SHELL181 elements are used for the mesh of stiffened plate models, considering welding residual stresses. In each case, 300 elements are used to model each local plate panel (the panel surrounded by successive longitudinal or transverse stiffeners), 6-7 and 5-6 elements are also considered, respectively, along web height and flange width of the stiffeners. Fig. 2 shows typical examples of the stiffener mesh models.

\subsection{Mechanical properties of materials}

The material properties were taken from the Aalberg experiments [13]. The Young's modulus and the Poisson ratio of the material are $70.475 \mathrm{GPa}$ and 0.3 , respectively. Stress-strain relationship of the aluminium alloy is shown in Fig. 3(a). The breadth of HAZ is assumed to be $50 \mathrm{~mm}$ in the plate and $25 \mathrm{~mm}$ in the stiffener web, at the plate-stiffener junction (Fig. 3(b)).

\subsection{Extent of the model, boundary and loading conditions}

A double span-single stiffener (DS-SS) or in other words double span-double bay (DS-DB) model (ABCD in Fig. 4) has been chosen

Table 1

Summary of the ultimate strengths of continuous stiffened aluminium plates obtained by FEA and developed empirical formulations (stiffener type: T-bar, lateral pressure: $H=0 \mathrm{~m})$.

\begin{tabular}{|c|c|c|c|c|c|c|c|c|c|c|c|c|}
\hline Stiffener type & ID & $a(\mathrm{~mm})$ & $b(\mathrm{~mm})$ & $t_{p}(\mathrm{~mm})$ & $h_{w}(\mathrm{~mm})$ & $t_{w}(\mathrm{~mm})$ & $b_{f}(\mathrm{~mm})$ & $t_{f}(\mathrm{~mm})$ & $\beta$ & $\lambda$ & $\left(\frac{\sigma_{u l t}}{\sigma_{Y S e q}}\right)_{F E M}$ & $\left(\frac{\sigma_{\text {ult }}}{\sigma_{\text {Yseq }}}\right)_{\text {formula }}$ \\
\hline 1 & 2 & 3 & 4 & 5 & 6 & 7 & 8 & 9 & 10 & 11 & 12 & 13 \\
\hline $\mathrm{T}$ & 1 & 1200 & 160 & 4 & 30 & 3 & 10 & 3 & 2.430 & 1.318 & 0.6239 & 0.5801 \\
\hline $\mathrm{T}$ & 2 & 1200 & 160 & 4 & 35 & 4 & 20 & 4 & 2.430 & 0.877 & 0.6958 & 0.6764 \\
\hline $\mathrm{T}$ & 3 & 1200 & 160 & 4 & 45 & 4 & 25 & 4 & 2.430 & 0.659 & 0.7197 & 0.7152 \\
\hline $\mathrm{T}$ & 4 & 1200 & 160 & 4 & 60 & 6 & 40 & 6 & 2.430 & 0.431 & 0.8007 & 0.7457 \\
\hline $\mathrm{T}$ & 5 & 1200 & 160 & 4 & 80 & 7 & 50 & 7 & 2.430 & 0.323 & 0.8284 & 0.7560 \\
\hline $\mathrm{T}$ & 6 & 1200 & 160 & 4 & 100 & 8 & 60 & 8 & 2.430 & 0.262 & 0.8554 & 0.7606 \\
\hline $\mathrm{T}$ & 7 & 1200 & 160 & 4 & 120 & 10 & 70 & 10 & 2.430 & 0.223 & 0.8626 & 0.7629 \\
\hline $\mathrm{T}$ & 8 & 1200 & 160 & 6 & 30 & 3 & 10 & 3 & 1.620 & 1.462 & 0.6621 & 0.6071 \\
\hline $\mathrm{T}$ & 9 & 1200 & 160 & 6 & 35 & 4 & 20 & 4 & 1.620 & 0.958 & 0.7681 & 0.7629 \\
\hline $\mathrm{T}$ & 10 & 1200 & 160 & 6 & 45 & 4 & 25 & 4 & 1.620 & 0.715 & 0.8476 & 0.8306 \\
\hline $\mathrm{T}$ & 11 & 1200 & 160 & 6 & 60 & 6 & 40 & 6 & 1.620 & 0.446 & 0.8928 & 0.8891 \\
\hline $\mathrm{T}$ & 12 & 1200 & 160 & 6 & 80 & 7 & 50 & 7 & 1.620 & 0.325 & 0.9112 & 0.9077 \\
\hline $\mathrm{T}$ & 13 & 1200 & 160 & 6 & 100 & 8 & 60 & 8 & 1.620 & 0.259 & 0.9247 & 0.9156 \\
\hline $\mathrm{T}$ & 14 & 1200 & 160 & 6 & 120 & 10 & 70 & 10 & 1.620 & 0.218 & 0.9395 & 0.9197 \\
\hline $\mathrm{T}$ & 15 & 1200 & 300 & 8 & 30 & 3 & 10 & 3 & 2.278 & 1.976 & 0.4004 & 0.4373 \\
\hline $\mathrm{T}$ & 16 & 1200 & 300 & 8 & 35 & 4 & 20 & 4 & 2.278 & 1.292 & 0.5865 & 0.6002 \\
\hline $\mathrm{T}$ & 17 & 1200 & 300 & 8 & 45 & 4 & 25 & 4 & 2.278 & 0.957 & 0.6736 & 0.6792 \\
\hline $\mathrm{T}$ & 18 & 1200 & 300 & 8 & 60 & 6 & 40 & 6 & 2.278 & 0.547 & 0.7843 & 0.7560 \\
\hline $\mathrm{T}$ & 19 & 1200 & 300 & 8 & 80 & 7 & 50 & 7 & 2.278 & 0.376 & 0.7854 & 0.7775 \\
\hline $\mathrm{T}$ & 20 & 1200 & 300 & 8 & 100 & 8 & 60 & 8 & 2.278 & 0.284 & 0.8182 & 0.7859 \\
\hline $\mathrm{T}$ & 21 & 1200 & 300 & 8 & 120 & 10 & 70 & 10 & 2.278 & 0.224 & 0.8495 & 0.7902 \\
\hline $\mathrm{T}$ & 22 & 1200 & 160 & 8 & 30 & 3 & 10 & 3 & 1.215 & 1.550 & 0.6648 & 0.6040 \\
\hline $\mathrm{T}$ & 23 & 1200 & 160 & 8 & 35 & 4 & 20 & 4 & 1.215 & 1.020 & 0.8122 & 0.7904 \\
\hline $\mathrm{T}$ & 24 & 1200 & 160 & 8 & 45 & 4 & 25 & 4 & 1.215 & 0.762 & 0.8250 & 0.8780 \\
\hline $\mathrm{T}$ & 25 & 1200 & 160 & 8 & 60 & 6 & 40 & 6 & 1.215 & 0.463 & 0.8867 & 0.9606 \\
\hline $\mathrm{T}$ & 26 & 1200 & 160 & 8 & 80 & 7 & 50 & 7 & 1.215 & 0.332 & 0.9220 & 0.9863 \\
\hline $\mathrm{T}$ & 27 & 1200 & 160 & 8 & 100 & 8 & 60 & 8 & 1.215 & 0.260 & 0.9310 & 0.9971 \\
\hline $\mathrm{T}$ & 28 & 1200 & 160 & 8 & 120 & 10 & 70 & 10 & 1.215 & 0.215 & 0.9475 & 1.0026 \\
\hline $\mathrm{T}$ & 29 & 1200 & 300 & 10 & 30 & 3 & 10 & 3 & 1.822 & 1.999 & 0.4472 & 0.4501 \\
\hline $\mathrm{T}$ & 30 & 1200 & 300 & 10 & 35 & 4 & 20 & 4 & 1.822 & 1.349 & 0.6335 & 0.6253 \\
\hline $\mathrm{T}$ & 31 & 1200 & 300 & 10 & 45 & 4 & 25 & 4 & 1.822 & 1.012 & 0.7509 & 0.7222 \\
\hline $\mathrm{T}$ & 32 & 1200 & 300 & 10 & 60 & 6 & 40 & 6 & 1.822 & 0.578 & 0.8782 & 0.8280 \\
\hline $\mathrm{T}$ & 33 & 1200 & 300 & 10 & 80 & 7 & 50 & 7 & 1.822 & 0.394 & 0.9039 & 0.8590 \\
\hline $\mathrm{T}$ & 34 & 1200 & 300 & 10 & 100 & 8 & 60 & 8 & 1.822 & 0.294 & 0.9172 & 0.8713 \\
\hline $\mathrm{T}$ & 35 & 1200 & 300 & 10 & 120 & 10 & 70 & 10 & 1.822 & 0.229 & 0.9364 & 0.8775 \\
\hline $\mathrm{T}$ & 36 & 900 & 300 & 5 & 30 & 4 & 20 & 4 & 3.644 & 1.988 & 0.3776 & 0.3760 \\
\hline $\mathrm{T}$ & 37 & 900 & 300 & 5 & 40 & 4 & 25 & 4 & 3.644 & 1.413 & 0.4611 & 0.4648 \\
\hline $\mathrm{T}$ & 38 & 900 & 300 & 5 & 45 & 5 & 30 & 5 & 3.644 & 1.105 & 0.5086 & 0.5090 \\
\hline $\mathrm{T}$ & 39 & 900 & 300 & 5 & 50 & 6 & 40 & 6 & 3.644 & 0.876 & 0.5378 & 0.5372 \\
\hline $\mathrm{T}$ & 40 & 900 & 300 & 5 & 80 & 7 & 50 & 7 & 3.644 & 0.520 & 0.6087 & 0.5701 \\
\hline $\mathrm{T}$ & 41 & 900 & 300 & 5 & 100 & 8 & 60 & 8 & 3.644 & 0.402 & 0.6255 & 0.5775 \\
\hline $\mathrm{T}$ & 42 & 900 & 300 & 6 & 40 & 4 & 20 & 4 & 3.037 & 1.580 & 0.4797 & 0.4781 \\
\hline $\mathrm{T}$ & 43 & 900 & 300 & 6 & 60 & 5 & 30 & 5 & 3.037 & 0.873 & 0.5597 & 0.6023 \\
\hline $\mathrm{T}$ & 44 & 900 & 300 & 6 & 80 & 8 & 40 & 8 & 3.037 & 0.542 & 0.6584 & 0.6431 \\
\hline
\end{tabular}


Table 2

Summary of the ultimate strengths of continuous stiffened aluminium plates obtained by FEA and developed empirical formulations (stiffener type: T-bar, lateral pressure: $H=5 \mathrm{~m})$.

\begin{tabular}{|c|c|c|c|c|c|c|c|c|c|c|c|c|}
\hline Stiffener type & ID & $a(\mathrm{~mm})$ & $b(\mathrm{~mm})$ & $t_{p}(\mathrm{~mm})$ & $h_{w}(\mathrm{~mm})$ & $t_{w}(\mathrm{~mm})$ & $b_{f}(\mathrm{~mm})$ & $t_{f}(\mathrm{~mm})$ & $\beta$ & $\lambda$ & $\left(\frac{\sigma_{u l t}}{\sigma_{\text {Yseq }}}\right)_{F E M}$ & $\left(\frac{\sigma_{\text {ult }}}{\sigma_{\text {Yseq }}}\right)_{\text {formula }}$ \\
\hline 1 & 2 & 3 & 4 & 5 & 6 & 7 & 8 & 9 & 10 & 11 & 12 & 13 \\
\hline $\mathrm{T}$ & 2 & 1200 & 160 & 4 & 35 & 4 & 20 & 4 & 2.43 & 0.88 & 0.6368 & 0.6164 \\
\hline $\mathrm{T}$ & 3 & 1200 & 160 & 4 & 45 & 4 & 25 & 4 & 2.43 & 0.66 & 0.6131 & 0.6931 \\
\hline $\mathrm{T}$ & 4 & 1200 & 160 & 4 & 60 & 6 & 40 & 6 & 2.43 & 0.43 & 0.7519 & 0.7668 \\
\hline $\mathrm{T}$ & 12 & 1200 & 160 & 6 & 80 & 7 & 50 & 7 & 1.62 & 0.33 & 0.8996 & 0.8751 \\
\hline $\mathrm{T}$ & 13 & 1200 & 160 & 6 & 100 & 8 & 60 & 8 & 1.62 & 0.26 & 0.9149 & 0.8856 \\
\hline $\mathrm{T}$ & 14 & 1200 & 160 & 6 & 120 & 10 & 70 & 10 & 1.62 & 0.22 & 0.9302 & 0.8910 \\
\hline $\mathrm{T}$ & 17 & 1200 & 300 & 8 & 45 & 4 & 25 & 4 & 2.28 & 0.96 & 0.6061 & 0.6078 \\
\hline $\mathrm{T}$ & 18 & 1200 & 300 & 8 & 60 & 6 & 40 & 6 & 2.28 & 0.55 & 0.7736 & 0.7486 \\
\hline $\mathrm{T}$ & 19 & 1200 & 300 & 8 & 80 & 7 & 50 & 7 & 2.28 & 0.38 & 0.7629 & 0.7983 \\
\hline $\mathrm{T}$ & 26 & 1200 & 160 & 8 & 80 & 7 & 50 & 7 & 1.21 & 0.33 & 0.9122 & 0.9086 \\
\hline $\mathrm{T}$ & 27 & 1200 & 160 & 8 & 100 & 8 & 60 & 8 & 1.21 & 0.26 & 0.9226 & 0.9176 \\
\hline $\mathrm{T}$ & 28 & 1200 & 160 & 8 & 120 & 10 & 70 & 10 & 1.21 & 0.22 & 0.9403 & 0.9222 \\
\hline $\mathrm{T}$ & 29 & 1200 & 300 & 10 & 30 & 3 & 10 & 3 & 1.82 & 2 & 0.3625 & 0.3609 \\
\hline $\mathrm{T}$ & 30 & 1200 & 300 & 10 & 35 & 4 & 20 & 4 & 1.82 & 1.35 & 0.5596 & 0.5353 \\
\hline $\mathrm{T}$ & 31 & 1200 & 300 & 10 & 45 & 4 & 25 & 4 & 1.82 & 1.01 & 0.6567 & 0.6486 \\
\hline $\mathrm{T}$ & 36 & 900 & 300 & 5 & 30 & 4 & 20 & 4 & 3.64 & 1.99 & 0.2492 & 0.2486 \\
\hline $\mathrm{T}$ & 37 & 900 & 300 & 5 & 40 & 4 & 25 & 4 & 3.64 & 1.41 & 0.3475 & 0.3444 \\
\hline $\mathrm{T}$ & 38 & 900 & 300 & 5 & 45 & 5 & 30 & 5 & 3.64 & 1.1 & 0.4370 & 0.4180 \\
\hline $\mathrm{T}$ & 42 & 900 & 300 & 6 & 40 & 4 & 20 & 4 & 3.04 & 1.58 & 0.3395 & 0.3542 \\
\hline $\mathrm{T}$ & 43 & 900 & 300 & 6 & 60 & 5 & 30 & 5 & 3.04 & 0.87 & 0.5304 & 0.5469 \\
\hline
\end{tabular}

Table 3

Summary of the ultimate strengths of continuous stiffened aluminium plates obtained by FEA and developed empirical formulations (stiffener type: T-bar, lateral pressure: $H=10 \mathrm{~m})$.

\begin{tabular}{|c|c|c|c|c|c|c|c|c|c|c|c|c|}
\hline Stiffener type & ID & $a(\mathrm{~mm})$ & $b(\mathrm{~mm})$ & $t_{p}(\mathrm{~mm})$ & $h_{w}(\mathrm{~mm})$ & $t_{w}(\mathrm{~mm})$ & $b_{f}(\mathrm{~mm})$ & $t_{f}(\mathrm{~mm})$ & $\beta$ & $\lambda$ & $\left(\frac{\sigma_{\text {ult }}}{\sigma_{\text {Yseq }}}\right)_{F E M}$ & $\left(\frac{\sigma_{u l t}}{\sigma_{\text {Yseq }}}\right)_{\text {formula }}$ \\
\hline 1 & 2 & 3 & 4 & 5 & 6 & 7 & 8 & 9 & 10 & 11 & 12 & 13 \\
\hline $\mathrm{T}$ & 2 & 1200 & 160 & 4 & 35 & 4 & 20 & 4 & 2.43 & 0.88 & 0.5762 & 0.5773 \\
\hline $\mathrm{T}$ & 4 & 1200 & 160 & 4 & 60 & 6 & 40 & 6 & 2.43 & 0.43 & 0.7046 & 0.8108 \\
\hline $\mathrm{T}$ & 12 & 1200 & 160 & 6 & 80 & 7 & 50 & 7 & 1.62 & 0.33 & 0.8820 & 0.9102 \\
\hline $\mathrm{T}$ & 13 & 1200 & 160 & 6 & 100 & 8 & 60 & 8 & 1.62 & 0.26 & 0.8963 & 0.8965 \\
\hline $\mathrm{T}$ & 14 & 1200 & 160 & 6 & 120 & 10 & 70 & 10 & 1.62 & 0.22 & 0.9124 & 0.8889 \\
\hline $\mathrm{T}$ & 17 & 1200 & 300 & 8 & 45 & 4 & 25 & 4 & 2.28 & 0.96 & 0.5441 & 0.5607 \\
\hline $\mathrm{T}$ & 18 & 1200 & 300 & 8 & 60 & 6 & 40 & 6 & 2.28 & 0.55 & 0.7490 & 0.7966 \\
\hline $\mathrm{T}$ & 19 & 1200 & 300 & 8 & 80 & 7 & 50 & 7 & 2.28 & 0.38 & 0.7470 & 0.8450 \\
\hline $\mathrm{T}$ & 26 & 1200 & 160 & 8 & 80 & 7 & 50 & 7 & 1.21 & 0.33 & 0.8966 & 0.9426 \\
\hline $\mathrm{T}$ & 27 & 1200 & 160 & 8 & 100 & 8 & 60 & 8 & 1.21 & 0.26 & 0.9091 & 0.9147 \\
\hline $\mathrm{T}$ & 28 & 1200 & 160 & 8 & 120 & 10 & 70 & 10 & 1.21 & 0.22 & 0.9297 & 0.9005 \\
\hline $\mathrm{T}$ & 30 & 1200 & 300 & 10 & 35 & 4 & 20 & 4 & 1.82 & 1.35 & 0.4905 & 0.4048 \\
\hline $\mathrm{T}$ & 31 & 1200 & 300 & 10 & 45 & 4 & 25 & 4 & 1.82 & 1.01 & 0.5998 & 0.6387 \\
\hline $\mathrm{T}$ & 36 & 900 & 300 & 5 & 30 & 4 & 20 & 4 & 3.64 & 1.99 & 0.1397 & 0.1390 \\
\hline $\mathrm{T}$ & 37 & 900 & 300 & 5 & 40 & 4 & 25 & 4 & 3.64 & 1.41 & 0.2343 & 0.2274 \\
\hline $\mathrm{T}$ & 38 & 900 & 300 & 5 & 45 & 5 & 30 & 5 & 3.64 & 1.1 & 0.3801 & 0.3085 \\
\hline $\mathrm{T}$ & 42 & 900 & 300 & 6 & 40 & 4 & 20 & 4 & 3.04 & 1.58 & 0.2079 & 0.2232 \\
\hline $\mathrm{T}$ & 43 & 900 & 300 & 6 & 60 & 5 & 30 & 5 & 3.04 & 0.87 & 0.4987 & 0.4706 \\
\hline $\mathrm{T}$ & 44 & 900 & 300 & 6 & 80 & 8 & 40 & 8 & 3.04 & 0.54 & 0.6399 & 0.6606 \\
\hline
\end{tabular}

for analysis of the ultimate strength of stiffened aluminium plates with symmetrical stiffeners of either flat-bar or T-bar crosssections [30]. Boundary conditions of the analysed stiffened plates, as explained in Khedmati et al. [28], are as follows:

- symmetry conditions are imposed along longitudinal edges;

- symmetry conditions are imposed along transverse edges;

- although transverse frames are not modelled, the out-of-plane deformation of plate is restrained along its junction line with the transverse frame;

- to consider the plate continuity, in-plane movement of plate edges in their perpendicular directions is assumed to be uniform.

After producing initial deflections in the stiffened plate, lateral pressure is applied first. Then, longitudinal compression is applied on the stiffened plate.

\subsection{Initial imperfections}

In order to simulate the complex pattern of initial deflection [19], lateral pressure was applied first on the stiffened plate model and a linear elastic finite element analysis was carried out. Such an analysis was repeated in a trial and error sequence of calculations until the deflection of plate reached the following average value:

$W_{0 \text { max }}=c \beta^{2} t$

When this condition is satisfied, data informations, i.e. coordinates of nodal points, element coordinates and boundary conditions, were extracted and transferred to a new finite element mesh. The considered model is a non-linear FEM analysis of the stiffened plate subjected to in-plane compression with variable lateral loads. The procedure generating initial deflection is shown in Fig. 5. After this step, lateral pressure is applied until 
Table 4

Summary of the ultimate strengths of continuous stiffened aluminium plates obtained by FEA and developed empirical formulations (stiffener type: F-bar, lateral pressure: $H=0 \mathrm{~m})$.

\begin{tabular}{|c|c|c|c|c|c|c|c|c|c|c|c|c|}
\hline Stiffener type & ID & $a(\mathrm{~mm})$ & $b(\mathrm{~mm})$ & $t_{p}(\mathrm{~mm})$ & $h_{w}(\mathrm{~mm})$ & $t_{w}(\mathrm{~mm})$ & $b_{f}(\mathrm{~mm})$ & $t_{f}(\mathrm{~mm})$ & $\beta$ & $\lambda$ & $\left(\frac{\sigma_{u l t}}{\sigma_{\text {Yseq }}}\right)_{F E M}$ & $\left(\frac{\sigma_{\text {ult }}}{\sigma_{\text {Yseq }}}\right)_{\text {formula }}$ \\
\hline 1 & 2 & 3 & 4 & 5 & 6 & 7 & 8 & 9 & 10 & 11 & 12 & 13 \\
\hline $\mathrm{F}$ & 1 & 1200 & 160 & 10 & 40 & 4 & 0 & 0 & 0.9718 & 1.3734 & 0.7336 & 0.8164 \\
\hline $\mathrm{F}$ & 2 & 1200 & 160 & 10 & 65 & 5 & 0 & 0 & 0.9718 & 0.7144 & 0.8291 & 0.7873 \\
\hline F & 3 & 1200 & 160 & 10 & 80 & 5 & 0 & 0 & 0.9718 & 0.5547 & 0.8450 & 0.7994 \\
\hline $\mathrm{F}$ & 4 & 1200 & 160 & 10 & 95 & 6 & 0 & 0 & 0.9718 & 0.4277 & 0.8036 & 0.8093 \\
\hline $\mathrm{F}$ & 5 & 1200 & 300 & 10 & 40 & 4 & 0 & 0 & 1.8222 & 1.7350 & 0.5014 & 0.5309 \\
\hline $\mathrm{F}$ & 6 & 1200 & 300 & 10 & 50 & 4 & 0 & 0 & 1.8222 & 1.3525 & 0.5892 & 0.5529 \\
\hline $\mathrm{F}$ & 7 & 1200 & 300 & 10 & 90 & 6 & 0 & 0 & 1.8222 & 0.5574 & 0.7929 & 0.6931 \\
\hline $\mathrm{F}$ & 8 & 1200 & 300 & 10 & 110 & 6 & 0 & 0 & 1.8222 & 0.4321 & 0.7515 & 0.7174 \\
\hline $\mathrm{F}$ & 9 & 1200 & 300 & 10 & 130 & 8 & 0 & 0 & 1.8222 & 0.3211 & 0.7537 & 0.7360 \\
\hline F & 10 & 1200 & 300 & 8 & 40 & 4 & 0 & 0 & 2.2777 & 1.6906 & 0.4502 & 0.4234 \\
\hline $\mathrm{F}$ & 11 & 1200 & 300 & 8 & 80 & 5 & 0 & 0 & 2.2777 & 0.6507 & 0.6285 & 0.6109 \\
\hline $\mathrm{F}$ & 12 & 1200 & 300 & 8 & 95 & 6 & 0 & 0 & 2.2777 & 0.4902 & 0.6286 & 0.6504 \\
\hline $\mathrm{F}$ & 13 & 1200 & 300 & 8 & 110 & 6 & 0 & 0 & 2.2777 & 0.4075 & 0.6150 & 0.6693 \\
\hline $\mathrm{F}$ & 14 & 1200 & 300 & 8 & 130 & 8 & 0 & 0 & 2.2777 & 0.3064 & 0.6295 & 0.6897 \\
\hline $\mathrm{F}$ & 15 & 1200 & 300 & 6 & 65 & 5 & 0 & 0 & 3.0370 & 0.7827 & 0.5185 & 0.4831 \\
\hline F & 16 & 1200 & 300 & 6 & 80 & 5 & 0 & 0 & 3.0370 & 0.5995 & 0.5178 & 0.5335 \\
\hline $\mathrm{F}$ & 17 & 1200 & 300 & 6 & 95 & 6 & 0 & 0 & 3.0370 & 0.4554 & 0.5278 & 0.5736 \\
\hline F & 18 & 1200 & 300 & 6 & 110 & 6 & 0 & 0 & 3.0370 & 0.3798 & 0.5238 & 0.5935 \\
\hline $\mathrm{F}$ & 19 & 1200 & 300 & 6 & 130 & 8 & 0 & 0 & 3.0370 & 0.2909 & 0.5442 & 0.6145 \\
\hline $\mathrm{F}$ & 20 & 1200 & 160 & 8 & 40 & 4 & 0 & 0 & 1.2148 & 1.3268 & 0.7248 & 0.7214 \\
\hline $\mathrm{F}$ & 21 & 1200 & 160 & 8 & 65 & 5 & 0 & 0 & 1.2148 & 0.6804 & 0.8249 & 0.7567 \\
\hline $\mathrm{F}$ & 22 & 1200 & 160 & 8 & 80 & 5 & 0 & 0 & 1.2148 & 0.5283 & 0.7851 & 0.7747 \\
\hline $\mathrm{F}$ & 23 & 1200 & 160 & 8 & 95 & 6 & 0 & 0 & 1.2148 & 0.4099 & 0.7912 & 0.7878 \\
\hline F & 24 & 1200 & 160 & 6 & 80 & 5 & 0 & 0 & 1.6197 & 0.4979 & 0.7515 & 0.7301 \\
\hline $\mathrm{F}$ & 25 & 1200 & 160 & 6 & 95 & 6 & 0 & 0 & 1.6197 & 0.3915 & 0.7168 & 0.7473 \\
\hline $\mathrm{F}$ & 26 & 900 & 300 & 5 & 40 & 4 & 0 & 0 & 3.6444 & 1.1344 & 0.3283 & 0.3441 \\
\hline F & 27 & 900 & 300 & 5 & 50 & 4 & 0 & 0 & 3.6444 & 0.8497 & 0.4314 & 0.4062 \\
\hline $\mathrm{F}$ & 28 & 900 & 300 & 5 & 65 & 5 & 0 & 0 & 3.6444 & 0.5573 & 0.5310 & 0.4855 \\
\hline $\mathrm{F}$ & 29 & 900 & 300 & 5 & 80 & 5 & 0 & 0 & 3.6444 & 0.4275 & 0.5412 & 0.5224 \\
\hline $\mathrm{F}$ & 30 & 900 & 300 & 5 & 95 & 6 & 0 & 0 & 3.6444 & 0.3271 & 0.5661 & 0.5490 \\
\hline $\mathrm{F}$ & 31 & 900 & 300 & 5 & 110 & 6 & 0 & 0 & 3.6444 & 0.2736 & 0.5624 & 0.5616 \\
\hline $\mathrm{F}$ & 32 & 900 & 300 & 5 & 130 & 8 & 0 & 0 & 3.6444 & 0.2123 & 0.5890 & 0.5742 \\
\hline $\mathrm{F}$ & 33 & 900 & 300 & 7 & 80 & 5 & 0 & 0 & 2.6031 & 0.4697 & 0.6279 & 0.6172 \\
\hline $\mathrm{F}$ & 34 & 900 & 300 & 7 & 95 & 6 & 0 & 0 & 2.6031 & 0.3550 & 0.6510 & 0.6449 \\
\hline F & 35 & 900 & 300 & 7 & 110 & 6 & 0 & 0 & 2.6031 & 0.2955 & 0.6448 & 0.6575 \\
\hline $\mathrm{F}$ & 36 & 900 & 300 & 7 & 130 & 8 & 0 & 0 & 2.6031 & 0.2240 & 0.6583 & 0.6704 \\
\hline $\mathrm{F}$ & 37 & 900 & 300 & 8 & 50 & 4 & 0 & 0 & 2.2777 & 0.9677 & 0.4691 & 0.5352 \\
\hline $\mathrm{F}$ & 38 & 900 & 300 & 8 & 65 & 5 & 0 & 0 & 2.2777 & 0.6369 & 0.6877 & 0.6144 \\
\hline F & 39 & 900 & 300 & 8 & 80 & 5 & 0 & 0 & 2.2777 & 0.4880 & 0.6825 & 0.6509 \\
\hline $\mathrm{F}$ & 40 & 900 & 300 & 8 & 95 & 6 & 0 & 0 & 2.2777 & 0.3677 & 0.7053 & 0.6778 \\
\hline $\mathrm{F}$ & 41 & 900 & 300 & 8 & 110 & 6 & 0 & 0 & 2.2777 & 0.3056 & 0.6935 & 0.6899 \\
\hline F & 42 & 900 & 300 & 8 & 130 & 8 & 0 & 0 & 2.2777 & 0.2298 & 0.7030 & 0.7024 \\
\hline
\end{tabular}

the assumed levels, before the application of in-plane longitudinal compression load [31,32].

The average value of initial deflection is assumed to be as follows, based on the investigations made by Varghese [33]:

$W_{0 \max }=0.05 \beta^{2} t$

In addition to initial deflections in both plate and stiffener, material softening in the so-called heat-affected zone (HAZ) and also residual welding stresses are taken into account.

\subsection{Verification of code and approach}

Two of the models tested by Zha and Moan [17] were selected for verification purpose. Details of simulation activities and also obtained results are given in Khedmati et al. [28]. It was shown there that both the code and also adopted modelling scheme lead to accurate results in comparison with the test results obtained by Zha and Moan.

\section{Ultimate strength formulations}

\subsection{General form of the formulations}

Paik [23] derived closed-form empirical ultimate strength formulae for stiffened aluminium plate structures under axial compressive loads by regression analysis of experimental and numerical databases. The derived formulations by Paik [23] were as follows.

For the case of aluminium plate with T-bar stiffeners

$\frac{\sigma_{u l t}}{\sigma_{\text {Yseq }}}=\frac{1}{\sqrt{1.318+0.185 \beta^{2}+2.759 \lambda^{2}-0.177 \lambda^{2} \beta^{2}+1.003 \lambda^{4}}} \leq \frac{1}{\lambda^{2}}$

For the case of aluminium plate with flat-bar stiffeners

$\frac{\sigma_{u l t}}{\sigma_{\text {Yseq }}}=\min \left\{\begin{array}{c}\frac{1}{\sqrt{2.500+0.084 \beta^{2}-0.588 \lambda^{2}+0.069 \lambda^{2} \beta^{2}+1.217 \lambda^{4}}} \leq \frac{1}{\lambda^{2}}, \\ \frac{1}{\sqrt{-16.297+17.716 \beta+18.776 \lambda-22.507 \lambda \beta}}\end{array}\right.$ 
Table 5

Summary of the ultimate strengths of continuous stiffened aluminium plates obtained by FEA and developed empirical formulations (stiffener type: F-bar, lateral pressure: $H=5 \mathrm{~m})$.

\begin{tabular}{|c|c|c|c|c|c|c|c|c|c|c|c|c|}
\hline Stiffener type & ID & $a(\mathrm{~mm})$ & $b(\mathrm{~mm})$ & $t_{p}(\mathrm{~mm})$ & $h_{w}(\mathrm{~mm})$ & $t_{w}(\mathrm{~mm})$ & $b_{f}(\mathrm{~mm})$ & $t_{f}(\mathrm{~mm})$ & $\beta$ & $\lambda$ & $\left(\frac{\sigma_{\text {ults }}}{\sigma_{\text {Yseq }}}\right)_{F E M}$ & $\left(\frac{\sigma_{u t t}}{\sigma_{\text {Yseq }}}\right)_{f o r m u l a}$ \\
\hline 1 & 2 & 3 & 4 & 5 & 6 & 7 & 8 & 9 & 10 & 11 & 12 & 13 \\
\hline $\mathrm{F}$ & 1 & 1200 & 160 & 10 & 40 & 4 & 0 & 0 & 0.97183 & 1.37337 & 0.6558 & 0.7108 \\
\hline $\mathrm{F}$ & 2 & 1200 & 160 & 10 & 65 & 5 & 0 & 0 & 0.97183 & 0.71437 & 0.8229 & 0.8096 \\
\hline $\mathrm{F}$ & 3 & 1200 & 160 & 10 & 80 & 5 & 0 & 0 & 0.97183 & 0.55472 & 0.7857 & 0.8207 \\
\hline $\mathrm{F}$ & 4 & 1200 & 160 & 10 & 95 & 6 & 0 & 0 & 0.97183 & 0.427717 & 0.7920 & 0.8269 \\
\hline $\mathrm{F}$ & 5 & 1200 & 300 & 10 & 40 & 4 & 0 & 0 & 1.82217 & 1.73501 & 0.4181 & 0.4173 \\
\hline $\mathrm{F}$ & 6 & 1200 & 300 & 10 & 50 & 4 & 0 & 0 & 1.82217 & 1.35251 & 0.5400 & 0.4975 \\
\hline $\mathrm{F}$ & 7 & 1200 & 300 & 10 & 90 & 6 & 0 & 0 & 1.82217 & 0.55736 & 0.7704 & 0.6773 \\
\hline $\mathrm{F}$ & 8 & 1200 & 300 & 10 & 110 & 6 & 0 & 0 & 1.82217 & 0.432099 & 0.7471 & 0.6994 \\
\hline $\mathrm{F}$ & 9 & 1200 & 300 & 10 & 130 & 8 & 0 & 0 & 1.82217 & 0.321078 & 0.7520 & 0.7153 \\
\hline $\mathrm{F}$ & 10 & 1200 & 300 & 8 & 40 & 4 & 0 & 0 & 2.27772 & 1.69063 & 0.3537 & 0.3560 \\
\hline $\mathrm{F}$ & 11 & 1200 & 300 & 8 & 80 & 5 & 0 & 0 & 2.27772 & 0.650714 & 0.6123 & 0.5840 \\
\hline $\mathrm{F}$ & 12 & 1200 & 300 & 8 & 95 & 6 & 0 & 0 & 2.27772 & 0.490228 & 0.6235 & 0.6204 \\
\hline $\mathrm{F}$ & 13 & 1200 & 300 & 8 & 110 & 6 & 0 & 0 & 2.27772 & 0.40747 & 0.6120 & 0.6371 \\
\hline $\mathrm{F}$ & 14 & 1200 & 300 & 8 & 130 & 8 & 0 & 0 & 2.27772 & 0.30637 & 0.6305 & 0.6545 \\
\hline $\mathrm{F}$ & 15 & 1200 & 300 & 6 & 65 & 5 & 0 & 0 & 3.03696 & 0.782732 & 0.4840 & 0.4503 \\
\hline $\mathrm{F}$ & 16 & 1200 & 300 & 6 & 80 & 5 & 0 & 0 & 3.03696 & 0.59947 & 0.4966 & 0.4957 \\
\hline $\mathrm{F}$ & 17 & 1200 & 300 & 6 & 95 & 6 & 0 & 0 & 3.03696 & 0.455368 & 0.5147 & 0.5302 \\
\hline $\mathrm{F}$ & 18 & 1200 & 300 & 6 & 110 & 6 & 0 & 0 & 3.03696 & 0.379765 & 0.5128 & 0.5467 \\
\hline $\mathrm{F}$ & 19 & 1200 & 300 & 6 & 130 & 8 & 0 & 0 & 3.03696 & 0.29085 & 0.5420 & 0.5638 \\
\hline $\mathrm{F}$ & 20 & 1200 & 160 & 8 & 40 & 4 & 0 & 0 & 1.21478 & 1.32681 & 0.6368 & 0.6486 \\
\hline $\mathrm{F}$ & 21 & 1200 & 160 & 8 & 65 & 5 & 0 & 0 & 1.21478 & 0.680404 & 0.8116 & 0.7665 \\
\hline $\mathrm{F}$ & 22 & 1200 & 160 & 8 & 80 & 5 & 0 & 0 & 1.21478 & 0.52826 & 0.7726 & 0.7837 \\
\hline $\mathrm{F}$ & 23 & 1200 & 160 & 8 & 95 & 6 & 0 & 0 & 1.21478 & 0.40994 & 0.7805 & 0.7940 \\
\hline $\mathrm{F}$ & 24 & 1200 & 160 & 6 & 80 & 5 & 0 & 0 & 1.61971 & 0.497898 & 0.7204 & 0.7209 \\
\hline $\mathrm{F}$ & 25 & 1200 & 160 & 6 & 95 & 6 & 0 & 0 & 1.61971 & 0.391458 & 0.7170 & 0.7359 \\
\hline $\mathrm{F}$ & 26 & 900 & 300 & 5 & 65 & 5 & 0 & 0 & 3.644352 & 0.55732 & 0.4101 & 0.4432 \\
\hline $\mathrm{F}$ & 27 & 900 & 300 & 5 & 80 & 5 & 0 & 0 & 3.644352 & 0.427538 & 0.4637 & 0.4735 \\
\hline $\mathrm{F}$ & 28 & 900 & 300 & 5 & 95 & 6 & 0 & 0 & 3.644352 & 0.327112 & 0.5138 & 0.4946 \\
\hline $\mathrm{F}$ & 29 & 900 & 300 & 5 & 110 & 6 & 0 & 0 & 3.644352 & 0.27362 & 0.5189 & 0.5044 \\
\hline $\mathrm{F}$ & 30 & 900 & 300 & 5 & 130 & 8 & 0 & 0 & 3.644352 & 0.212297 & 0.5658 & 0.5140 \\
\hline $\mathrm{F}$ & 31 & 900 & 300 & 7 & 80 & 5 & 0 & 0 & 2.6031 & 0.46973 & 0.5601 & 0.5801 \\
\hline $\mathrm{F}$ & 32 & 900 & 300 & 7 & 95 & 6 & 0 & 0 & 2.6031 & 0.355049 & 0.6175 & 0.6038 \\
\hline $\mathrm{F}$ & 33 & 900 & 300 & 7 & 110 & 6 & 0 & 0 & 2.6031 & 0.295502 & 0.6204 & 0.6143 \\
\hline $\mathrm{F}$ & 34 & 900 & 300 & 7 & 130 & 8 & 0 & 0 & 2.6031 & 0.224007 & 0.6488 & 0.6248 \\
\hline $\mathrm{F}$ & 35 & 900 & 300 & 8 & 65 & 5 & 0 & 0 & 2.27772 & 0.636873 & 0.5057 & 0.5873 \\
\hline $\mathrm{F}$ & 36 & 900 & 300 & 8 & 80 & 5 & 0 & 0 & 2.27772 & 0.488035 & 0.5953 & 0.6209 \\
\hline $\mathrm{F}$ & 37 & 900 & 300 & 8 & 95 & 6 & 0 & 0 & 2.27772 & 0.367671 & 0.6734 & 0.6444 \\
\hline $\mathrm{F}$ & 38 & 900 & 300 & 8 & 110 & 6 & 0 & 0 & 2.27772 & 0.305604 & 0.6728 & 0.6546 \\
\hline $\mathrm{F}$ & 39 & 900 & 300 & 8 & 130 & 8 & 0 & 0 & 2.27772 & 0.22978 & 0.6970 & 0.6650 \\
\hline
\end{tabular}

where

$\beta=\frac{b}{t} \sqrt{\frac{\sigma_{Y p}}{E}}$

$\lambda=\frac{a}{\pi r} \sqrt{\frac{\sigma_{Y s e q}}{E}}$

$r=\sqrt{\frac{I}{b t+h_{w} t_{w}+b_{f} t_{f}}}$

$$
\begin{aligned}
I= & \frac{b t^{3}}{12}+b t\left(z_{0}-\frac{t}{2}\right)^{2}+\frac{h_{w}^{3} t_{w}}{12}+h_{w} t_{w}\left(z_{0}-t-\frac{h_{w}}{2}\right)^{2} \\
& +\frac{b_{f} t_{f}^{3}}{12}+b_{f} t_{f}\left(t+h_{w} t_{w}+\frac{t_{f}}{2}+z_{0}\right)^{2}
\end{aligned}
$$

$$
z_{0}=\frac{0.5 b t^{2}+h_{w} t_{w}\left(t+0.5 h_{w}\right)+b_{f} t_{f}\left(t+h_{w}+0.5 t_{f}\right)}{b t+h_{w} t_{w}+b_{f} t_{f}}
$$

$\sigma_{Y s e q}=\frac{\sigma_{Y p} b t+\sigma_{Y s}\left(h_{w} t_{w}+b_{f} t_{f}\right)}{b t+h_{w} t_{w}+b_{f} t_{f}}$

In this paper, the same form of ultimate strength formulation as described by Eq. (3) is used. Different coefficients are derived based on the developed numerical database in order to estimate the ultimate strength of stiffened aluminium plates with various types of stiffener cross-sections under in-plane compression alone or in combination with lateral load.

\subsection{Aluminium plates stiffened with T-bar stiffeners under in-plane axial compression}

The geometrical characteristics of the analyzed stiffened plates are given in the first 11 columns of Table 1 . The models are subjected to pure in-plane compression. Complete paths of averaged stress vs. averaged strain relationships are obtained using FEM. Fig. 6 shows the averaged stress vs. averaged strain curves for stiffened aluminium plates under pure in-plane compression. The curves are grouped based on values of slenderness parameter of the plate part in the stiffened plate models. Different trends are observed before reaching the ultimate strength level and thereafter. This issue is not addressed in this paper, but the reader is referred to Khedmati et al. [28]. The ultimate strengths of all models are extracted out of the averaged stress vs. averaged strain curves and inserted in column 12 of Table 1 .

Regression analysis (see the Appendix) is applied to the obtained numerical data in Table 1. For this purpose, the algorithm explained in the Appendix is programmed in MATLAB environment [34]. The same form of ultimate strength formulation as 
Table 6

Summary of the ultimate strengths of continuous stiffened aluminium plates obtained by FEA and developed empirical formulations (stiffener type: F-bar, lateral pressure: $H=10 \mathrm{~m})$.

\begin{tabular}{|c|c|c|c|c|c|c|c|c|c|c|c|c|}
\hline Stiffener type & ID & $a(\mathrm{~mm})$ & $b(\mathrm{~mm})$ & $t_{p}(\mathrm{~mm})$ & $h_{w}(\mathrm{~mm})$ & $t_{w}(\mathrm{~mm})$ & $b_{f}(\mathrm{~mm})$ & $t_{f}(\mathrm{~mm})$ & $\beta$ & $\lambda$ & $\left(\frac{\sigma_{u l t}}{\sigma_{Y s e q}}\right)_{F E M}$ & $\left(\frac{\sigma_{\text {ult }}}{\sigma_{\text {Yseq }}}\right)_{\text {formula }}$ \\
\hline 1 & 2 & 3 & 4 & 5 & 6 & 7 & 8 & 9 & 10 & 11 & 12 & 13 \\
\hline $\mathrm{F}$ & 1 & 1200 & 160 & 10 & 65 & 5 & 0 & 0 & 0.97183 & 0.71437 & 0.8017 & 0.8978 \\
\hline $\mathrm{F}$ & 2 & 1200 & 160 & 10 & 80 & 5 & 0 & 0 & 0.97183 & 0.55472 & 0.7783 & 0.9030 \\
\hline $\mathrm{F}$ & 3 & 1200 & 160 & 10 & 95 & 6 & 0 & 0 & 0.97183 & 0.427717 & 0.8622 & 0.9057 \\
\hline $\mathrm{F}$ & 4 & 1200 & 300 & 10 & 40 & 4 & 0 & 0 & 1.82217 & 1.73501 & 0.3368 & 0.3022 \\
\hline $\mathrm{F}$ & 5 & 1200 & 300 & 10 & 50 & 4 & 0 & 0 & 1.82217 & 1.35251 & 0.4325 & 0.3725 \\
\hline $\mathrm{F}$ & 6 & 1200 & 300 & 10 & 90 & 6 & 0 & 0 & 1.82217 & 0.55736 & 0.7505 & 0.6248 \\
\hline $\mathrm{F}$ & 7 & 1200 & 300 & 10 & 110 & 6 & 0 & 0 & 1.82217 & 0.432099 & 0.7387 & 0.6755 \\
\hline $\mathrm{F}$ & 8 & 1200 & 300 & 10 & 130 & 8 & 0 & 0 & 1.82217 & 0.321078 & 0.7480 & 0.7176 \\
\hline $\mathrm{F}$ & 9 & 1200 & 300 & 8 & 40 & 4 & 0 & 0 & 2.27772 & 1.69063 & 0.2250 & 0.2399 \\
\hline F & 10 & 1200 & 300 & 8 & 80 & 5 & 0 & 0 & 2.27772 & 0.650714 & 0.5944 & 0.4874 \\
\hline F & 11 & 1200 & 300 & 8 & 95 & 6 & 0 & 0 & 2.27772 & 0.490228 & 0.6144 & 0.5556 \\
\hline $\mathrm{F}$ & 12 & 1200 & 300 & 8 & 110 & 6 & 0 & 0 & 2.27772 & 0.40747 & 0.6065 & 0.5927 \\
\hline $\mathrm{F}$ & 13 & 1200 & 300 & 8 & 130 & 8 & 0 & 0 & 2.27772 & 0.30637 & 0.6308 & 0.6370 \\
\hline $\mathrm{F}$ & 14 & 1200 & 300 & 6 & 80 & 5 & 0 & 0 & 3.03696 & 0.59947 & 0.4718 & 0.3959 \\
\hline $\mathrm{F}$ & 15 & 1200 & 300 & 6 & 95 & 6 & 0 & 0 & 3.03696 & 0.455368 & 0.4983 & 0.4555 \\
\hline $\mathrm{F}$ & 16 & 1200 & 300 & 6 & 110 & 6 & 0 & 0 & 3.03696 & 0.379765 & 0.4994 & 0.4897 \\
\hline $\mathrm{F}$ & 17 & 1200 & 300 & 6 & 130 & 8 & 0 & 0 & 3.03696 & 0.29085 & 0.5361 & 0.5301 \\
\hline $\mathrm{F}$ & 18 & 1200 & 160 & 8 & 40 & 4 & 0 & 0 & 1.21478 & 1.32681 & 0.5521 & 0.6140 \\
\hline F & 19 & 1200 & 160 & 8 & 65 & 5 & 0 & 0 & 1.21478 & 0.680404 & 0.7864 & 0.7819 \\
\hline $\mathrm{F}$ & 20 & 1200 & 160 & 8 & 80 & 5 & 0 & 0 & 1.21478 & 0.52826 & 0.7608 & 0.8158 \\
\hline $\mathrm{F}$ & 21 & 1200 & 160 & 8 & 95 & 6 & 0 & 0 & 1.21478 & 0.40994 & 0.7642 & 0.8382 \\
\hline $\mathrm{F}$ & 22 & 1200 & 160 & 6 & 80 & 5 & 0 & 0 & 1.61971 & 0.497898 & 0.7051 & 0.7007 \\
\hline $\mathrm{F}$ & 23 & 1200 & 160 & 6 & 95 & 6 & 0 & 0 & 1.61971 & 0.391458 & 0.7012 & 0.7382 \\
\hline $\mathrm{F}$ & 24 & 900 & 300 & 5 & 80 & 5 & 0 & 0 & 3.644352 & 0.427538 & 0.3878 & 0.4014 \\
\hline F & 25 & 900 & 300 & 5 & 95 & 6 & 0 & 0 & 3.644352 & 0.327112 & 0.4627 & 0.4450 \\
\hline $\mathrm{F}$ & 26 & 900 & 300 & 5 & 110 & 6 & 0 & 0 & 3.644352 & 0.27362 & 0.4772 & 0.4684 \\
\hline $\mathrm{F}$ & 27 & 900 & 300 & 5 & 130 & 8 & 0 & 0 & 3.644352 & 0.212297 & 0.5362 & 0.4937 \\
\hline $\mathrm{F}$ & 28 & 900 & 300 & 7 & 80 & 5 & 0 & 0 & 2.6031 & 0.46973 & 0.4652 & 0.5095 \\
\hline $\mathrm{F}$ & 29 & 900 & 300 & 7 & 95 & 6 & 0 & 0 & 2.6031 & 0.355049 & 0.5799 & 0.5620 \\
\hline $\mathrm{F}$ & 30 & 900 & 300 & 7 & 110 & 6 & 0 & 0 & 2.6031 & 0.295502 & 0.5918 & 0.5887 \\
\hline $\mathrm{F}$ & 31 & 900 & 300 & 7 & 130 & 8 & 0 & 0 & 2.6031 & 0.224007 & 0.6334 & 0.6183 \\
\hline $\mathrm{F}$ & 32 & 900 & 300 & 8 & 80 & 5 & 0 & 0 & 2.27772 & 0.488035 & 0.4883 & 0.5566 \\
\hline $\mathrm{F}$ & 33 & 900 & 300 & 8 & 95 & 6 & 0 & 0 & 2.27772 & 0.367671 & 0.6412 & 0.6105 \\
\hline $\mathrm{F}$ & 34 & 900 & 300 & 8 & 110 & 6 & 0 & 0 & 2.27772 & 0.305604 & 0.6503 & 0.6373 \\
\hline $\mathrm{F}$ & 35 & 900 & 300 & 8 & 130 & 8 & 0 & 0 & 2.27772 & 0.22978 & 0.6871 & 0.6670 \\
\hline
\end{tabular}
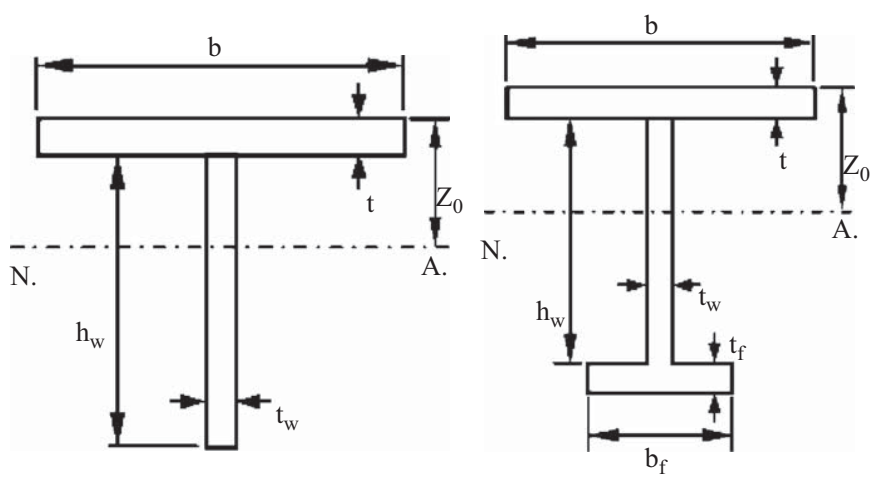

Fig. 1. Cross-sectional geometries of stiffened aluminium plates [23].

given by Eq. (3) is kept throughout the regression analysis. The coefficients are so updated to give suitable estimates of the ultimate strength for the case under consideration. The following equation is obtained as a result

$\frac{\sigma_{u l t}}{\sigma_{\text {Yseq }}}=\frac{1}{\sqrt{0.7318+0.1622 \beta^{2}+0.4833 \lambda^{2}+0.0148 \lambda^{2} \beta^{2}+0.0963 \lambda^{4}}} \leq \frac{1}{\lambda^{2}}$

Eq. (11) is used to predict the ultimate strengths of all models in Table 1. These values are placed in column 13 of Table 1 . In order to compare the results, non-dimensionalized ultimate strength values for aluminium plates stiffened by T-bar stiffeners a

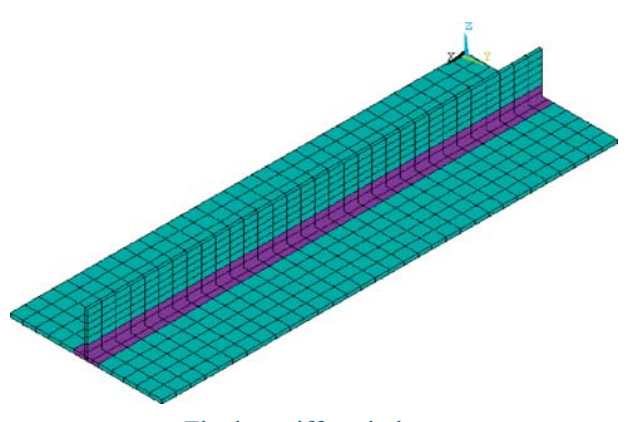

Flat bar stiffened plate

b

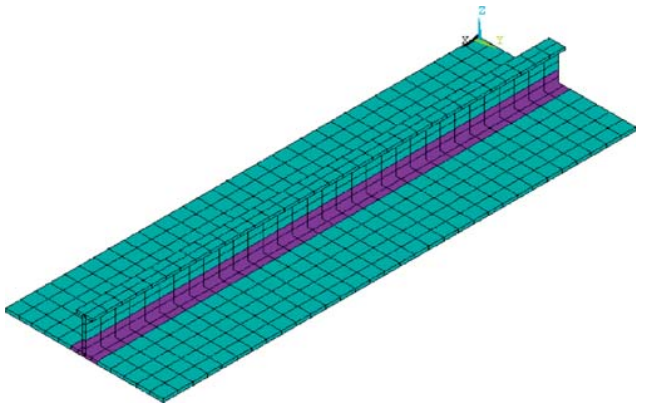

Tee bar stiffened plate

Fig. 2. Typical examples of the mesh models. 
a

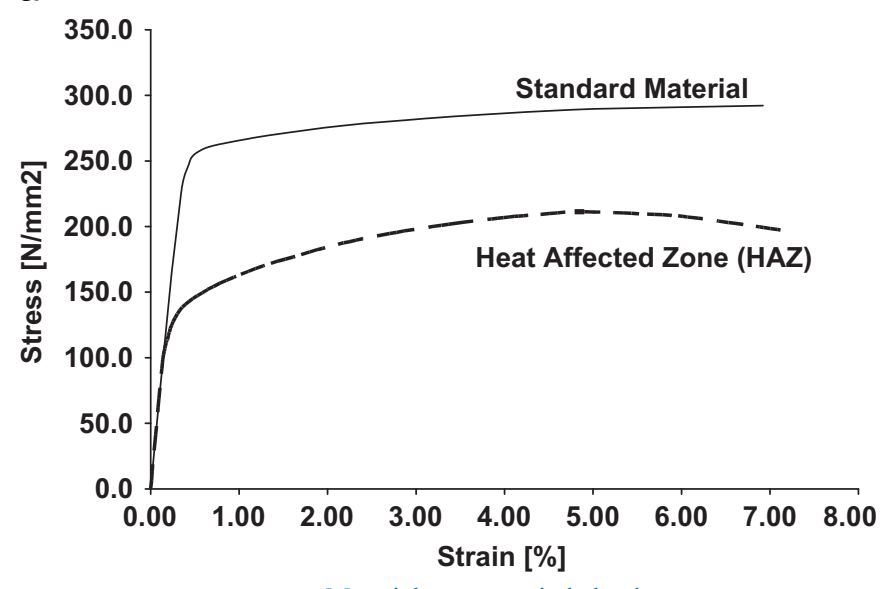

b

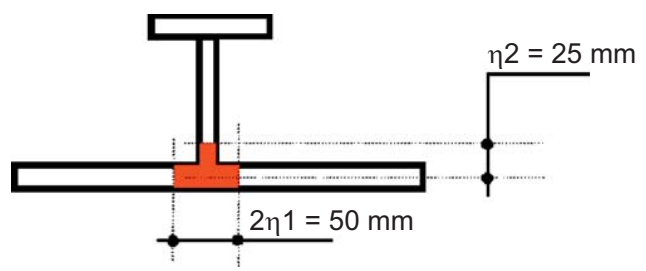

Extent of the heat affected zone (HAZ) in both plate and stiffener

Fig. 3. Stress-strain behaviour of the material and extent of the HAZ.
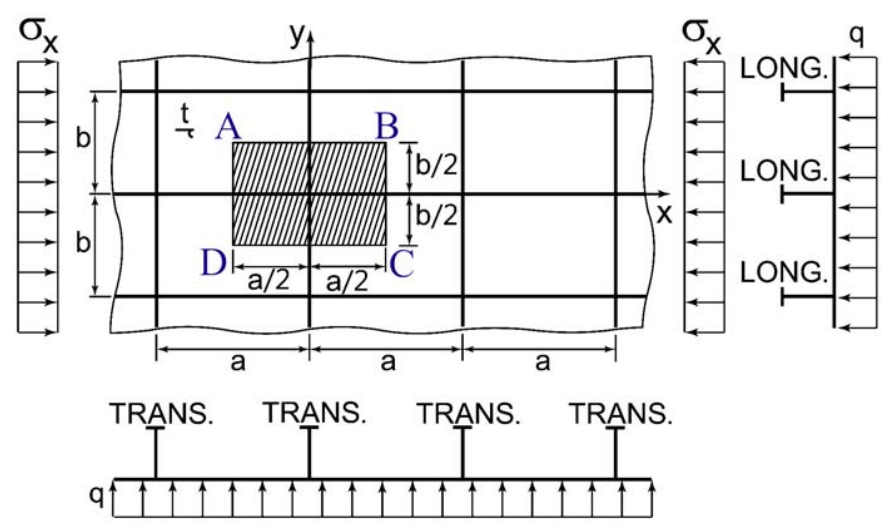

Fig. 4. Extent of the continuous stiffened plate models for analysis.

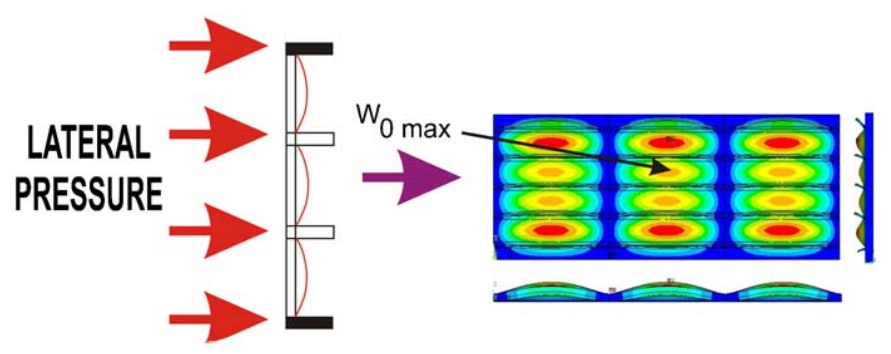

Fig. 5. Procedure to generate initial deflection.

(as indicated in Table 1) under pure in-plane axial compression are shown vs. their column slenderness parameters in Fig. 7. As observed, a good agreement is seen between FEM-based values of ultimate strengths and those predicted by Eq. (11). The favourable agreement is confirmed in Fig. 8, where a comparison of the nondimensionalised ultimate strength values for aluminium plates stiffened by T-bar stiffeners (as indicated in Table 1) under pure in-plane axial compression is shown.

3.3. Aluminium plates stiffened with T-bar stiffeners under combined in-plane axial compression and lateral pressure

In order to expand the range of empirical formulations for estimation of the ultimate strength of stiffened aluminium plates, some models are also analysed under combined in-plane compression and lateral pressure. Geometrical characteristics of the analysed stiffened plates are given in the first 11 columns of Tables 2 and 3. Two levels of water head $(h)$ are assumed: 5 and $10 \mathrm{~m}$. As was explained earlier in Section 2.4, after producing initial deflection in the stiffened plate, lateral pressure is applied first on it until assumed levels. Then, longitudinal compression is applied on the stiffened plate. Column number 12 in Tables 2 and 3 presents values of the ultimate strength of analysed stiffened aluminium plates under combined in-plane compression and lateral pressure, obtained by FEM.

A regression analysis, as explained in Section 3.2, is applied to the database of Tables 2 and 3. As a result, the following empirical equations (Eq. (12)) are derived in order to estimate the ultimate strength of stiffened aluminium plates under combined in-plane compression and lateral pressure.

For the case of aluminium plate with T-bar stiffeners under water head $=h=5 \mathrm{~m}$

$\frac{\sigma_{\text {ult }}}{\sigma_{\text {Yseq }}}=\frac{1}{\sqrt{1.0579+0.063 \beta^{2}+0.2298 \lambda^{2}+0.2028 \lambda^{2} \beta^{2}+0.1753 \lambda^{4}}} \leq \frac{1}{\lambda^{2}}$

For the case of aluminium plate with T-bar stiffeners under water head $=h=10 \mathrm{~m}$

$\frac{\sigma_{\text {ult }}}{\sigma_{\text {Yseq }}}=\frac{1}{\sqrt{1.3197+0.0011 \beta^{2} \lambda^{2}-2.8853 \lambda^{2}+0.6063 \lambda^{2} \beta^{2}+1.9248 \lambda^{4}}} \leq \frac{1}{\lambda^{2}}$

Predicted ultimate strengths using Eqs. (12) and (13) for the stiffened aluminium plates are given in column 13 of Tables 2 and 3. Also, FEM results and predictions of the ultimate strengths are compared with each other in Fig. 9. Good agreements are also observed in these cases.

\subsection{Aluminium plates stiffened with flat-bar stiffeners under combined in-plane axial compression and lateral pressure}

Studies made by Paik [23] showed that collapse characteristics of the aluminium plates with flat-bar stiffeners differ from those of the aluminium plates with T- or angle-bar stiffeners. Thus different ultimate strength formulations are needed to be developed for the case of the aluminium plates with flat-bar stiffeners. Accordingly, a set of stiffened aluminium plates having flat-bar stiffeners is designed and then analysed using FEM. Geometrical characteristics of the analysed stiffened plates are given in the first 11 columns of Tables 4-6. Values of ultimate strength for the models are given in column number 12 of those tables.

The same regression procedure is applied to the database of Tables 4-6. As a result, the following empirical equations (Eqs. (14)-(16)) are derived to estimate the ultimate strength of stiffened aluminium plates under combined in-plane compression and lateral pressure. 
a

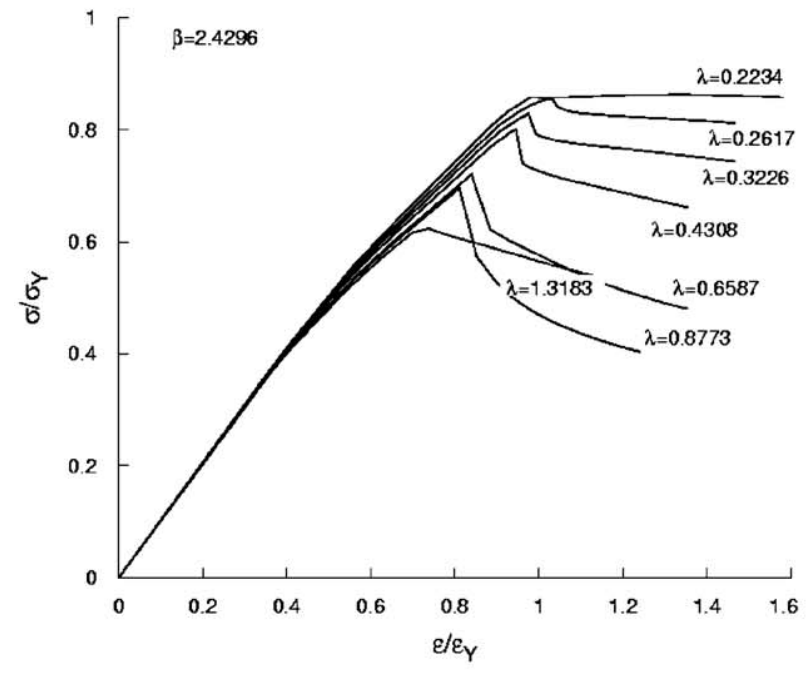

$\mathrm{T} 1-\mathrm{T} 7$

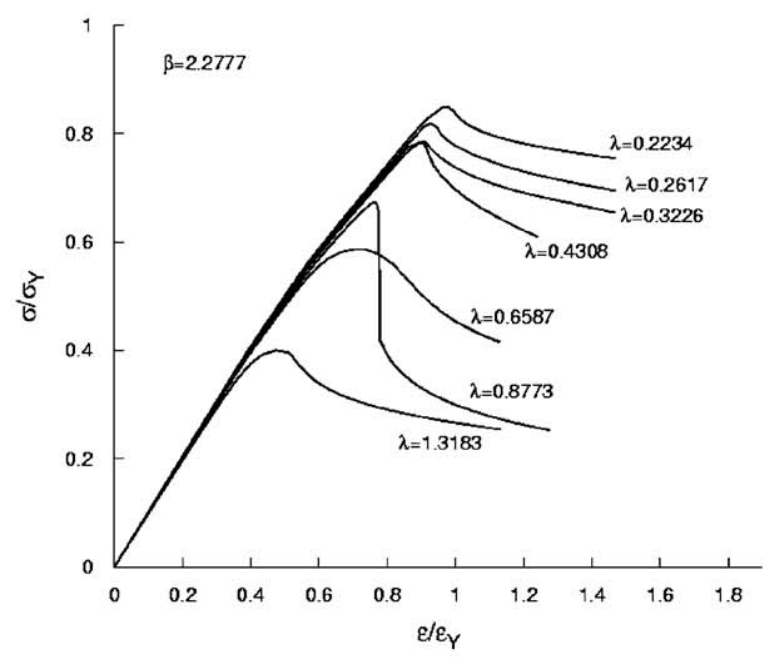

$\mathrm{T} 15-\mathrm{T} 21$

e

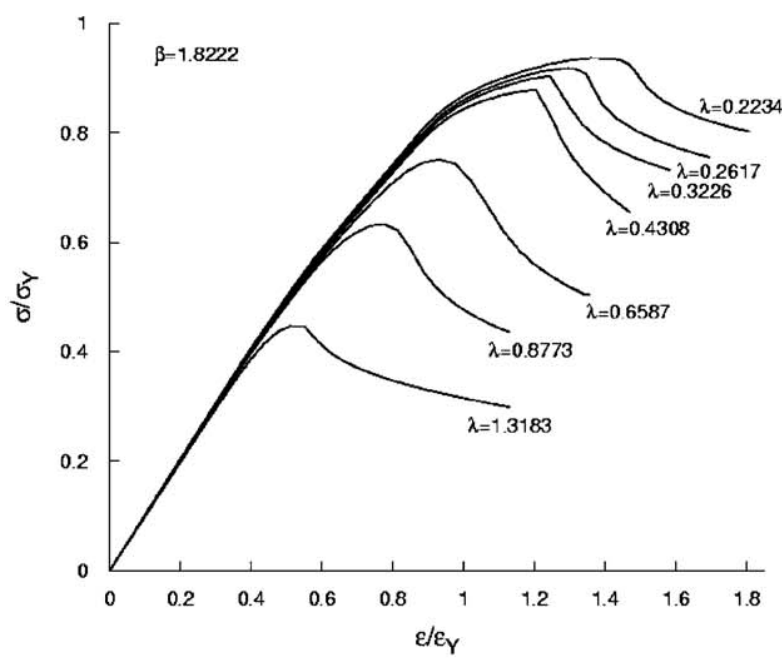

T29- T35 b

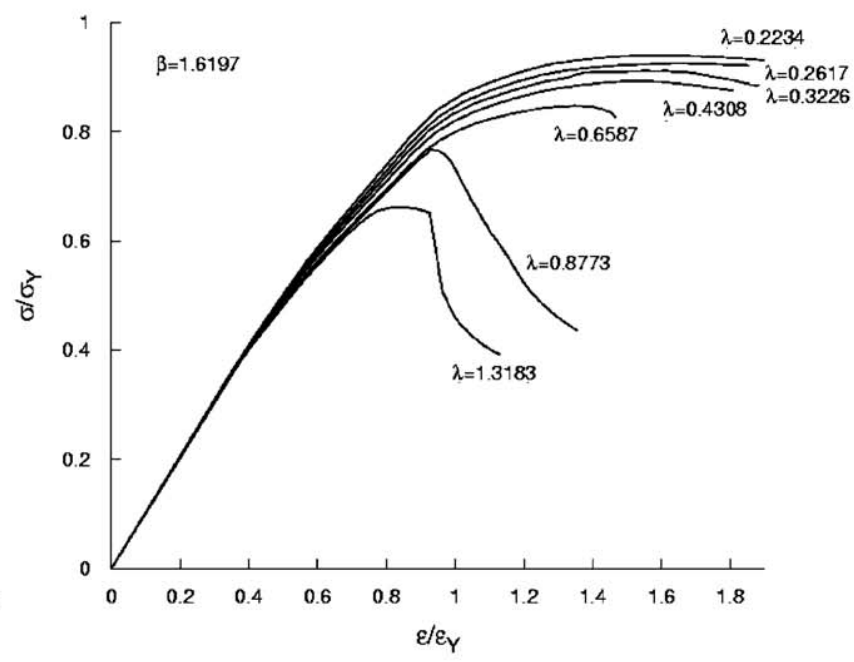

$\mathrm{T} 8-\mathrm{T} 14$

d

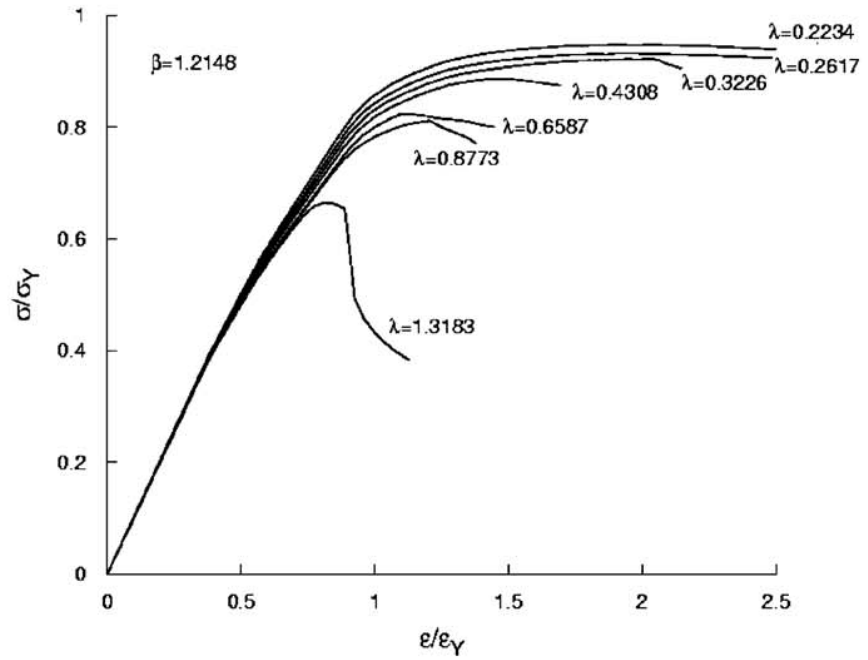

$\mathrm{T} 22-\mathrm{T} 28$

$f$

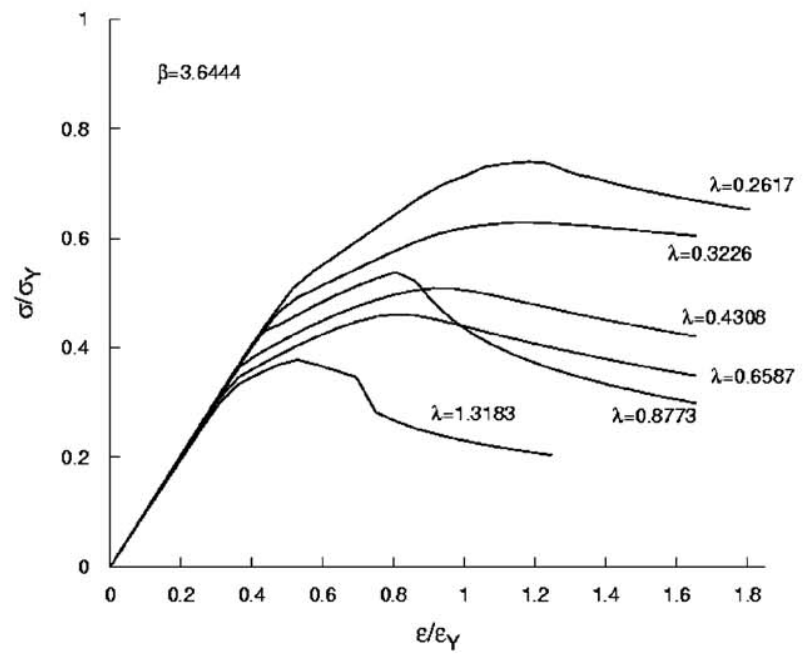

$\mathrm{T} 36-\mathrm{T} 41$

Fig. 6. Average stress-average strain curves for the aluminium plates stiffened with T-bar stiffeners (as indicated in Table 1) under pure in-plane axial compression. 
a

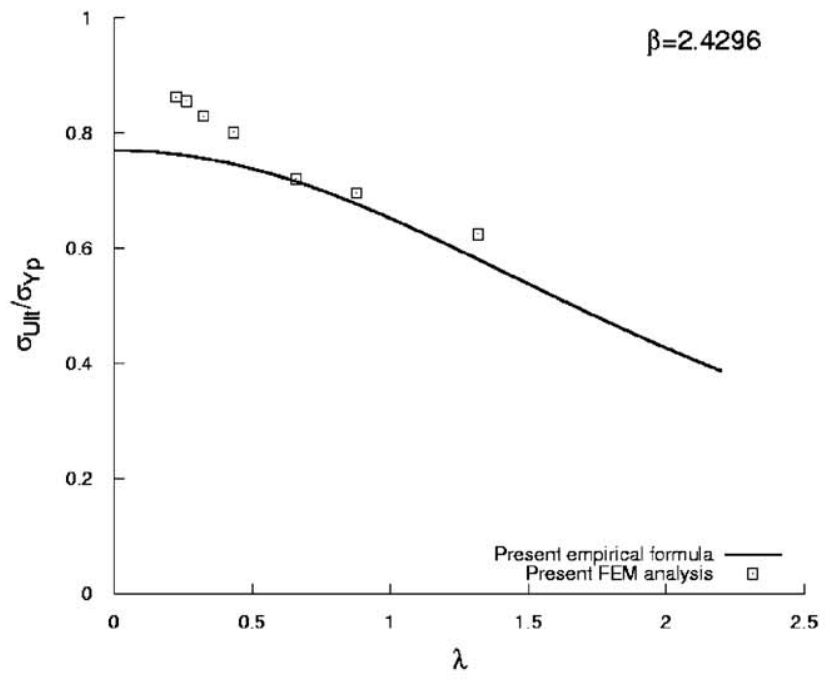

$\mathrm{T} 1-\mathrm{T} 7$

C

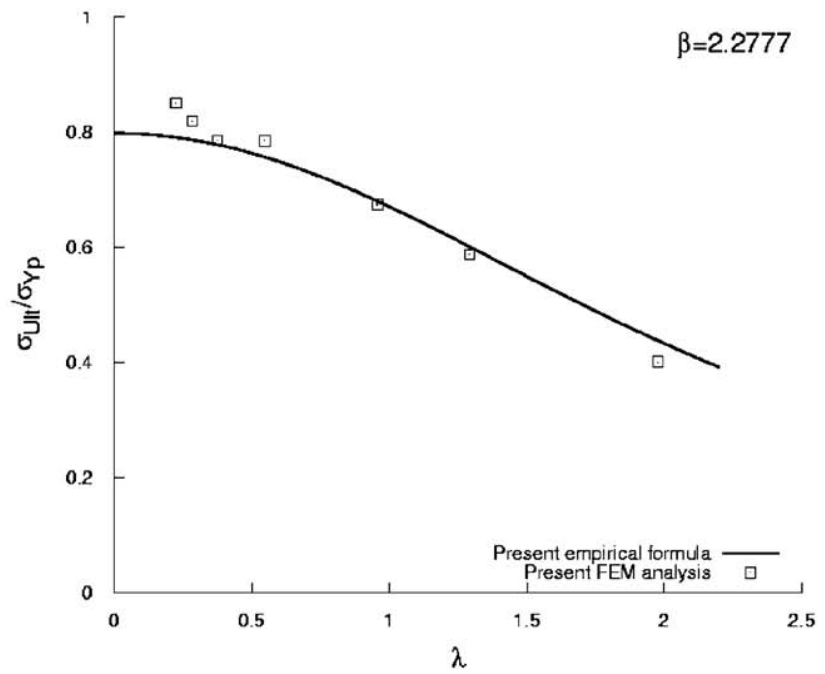

$\mathrm{T} 15-\mathrm{T} 21$

e

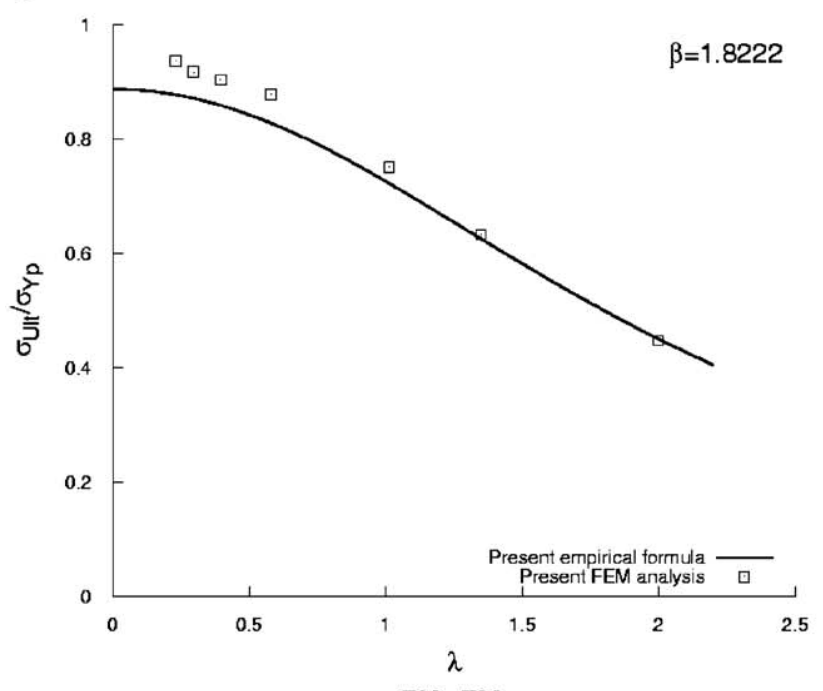

b

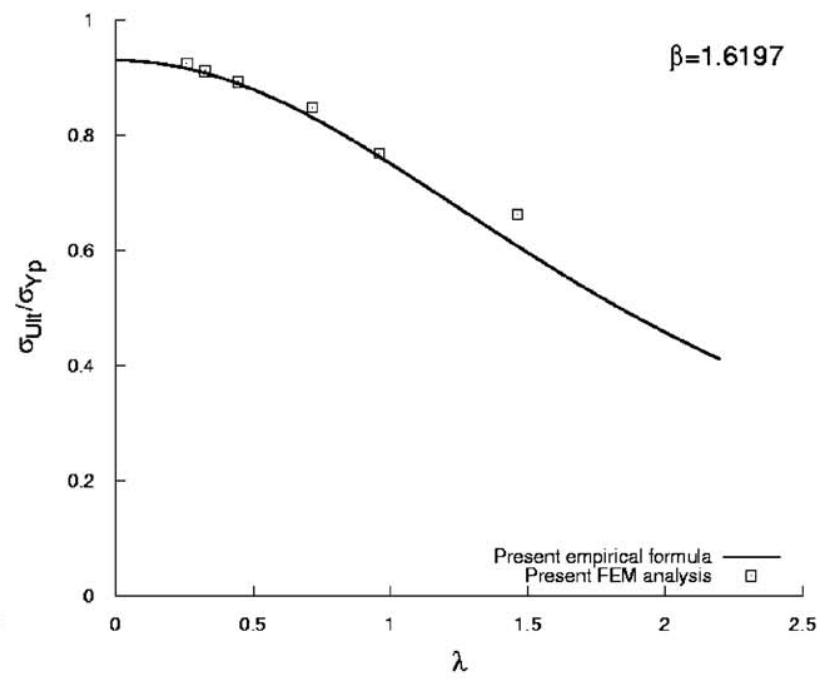

$\mathrm{T} 8-\mathrm{T} 14$

d

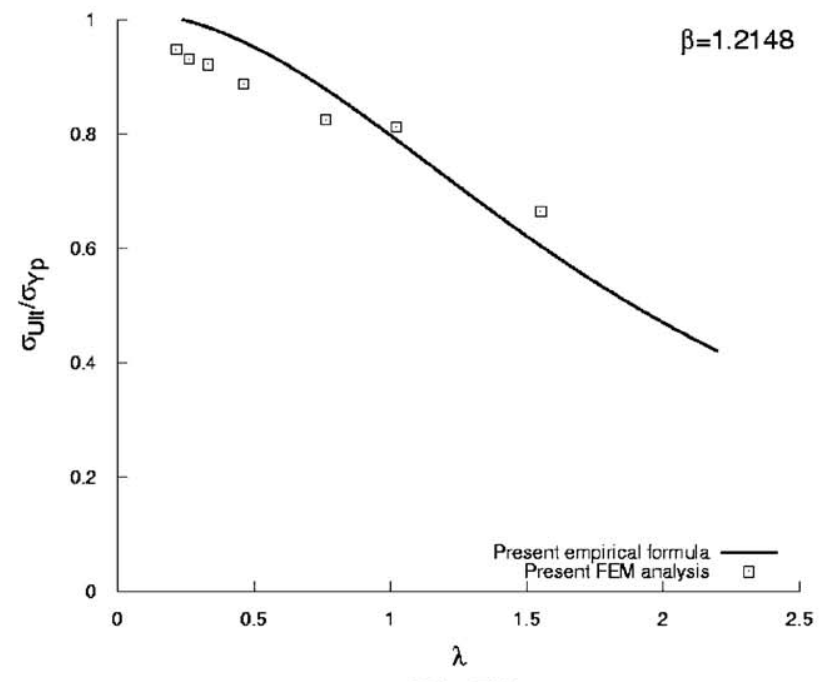

f

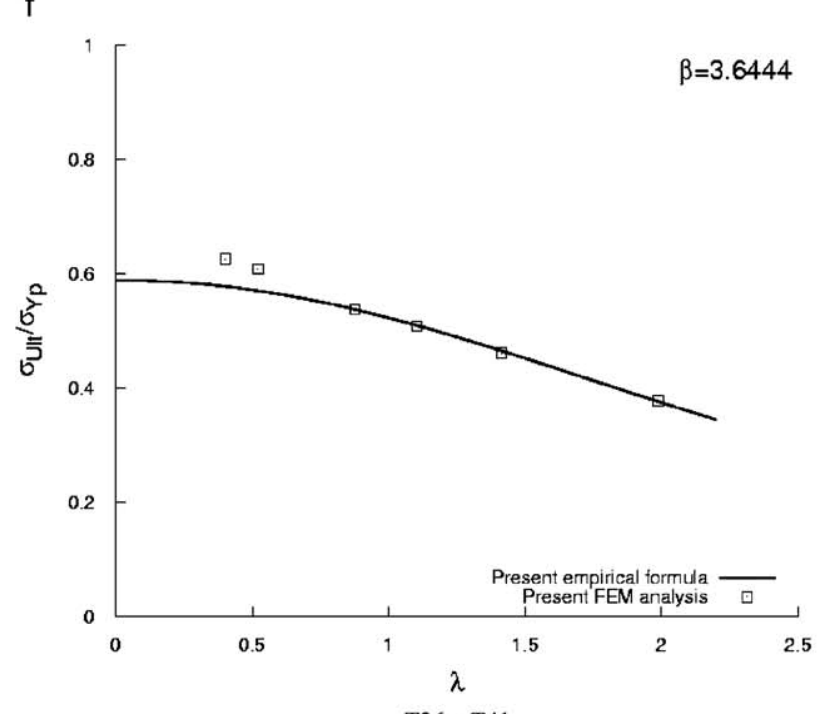

$\mathrm{T} 36-\mathrm{T} 41$

Fig. 7. Non-dimensionalised ultimate strength values for aluminium plates stiffened with T-bar stiffeners (as indicated in Table 1) under pure in-plane axial compression vs. their column slenderness parameters. 


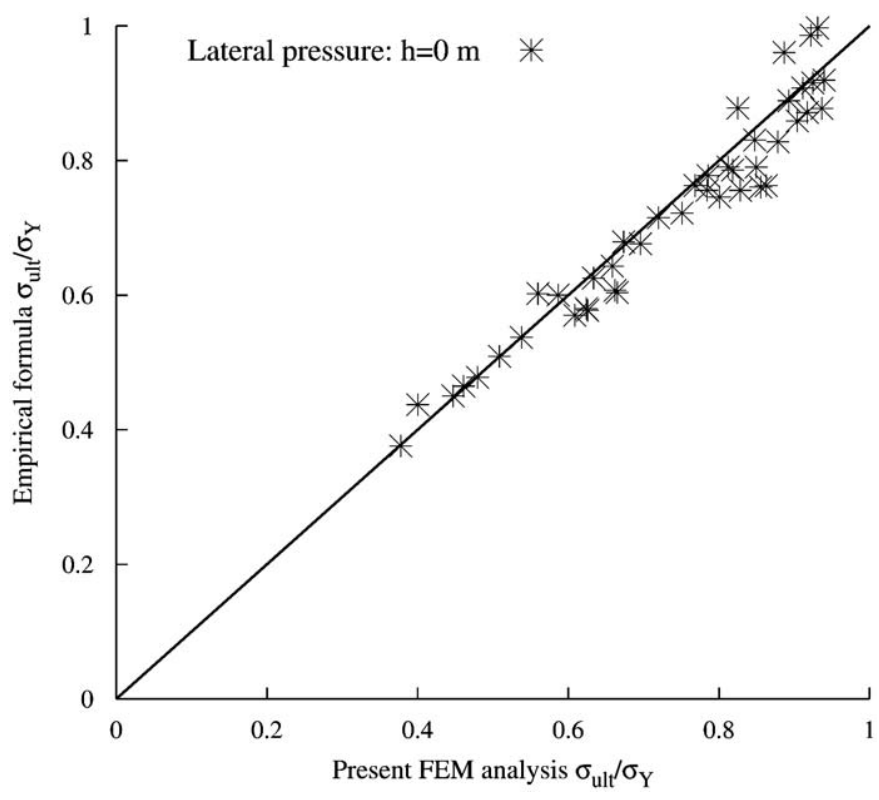

Fig. 8. Comparison of the non-dimensionalised ultimate strength values for aluminium plates stiffened with T-bar stiffeners (as indicated in Table 1) under pure in-plane axial compression.

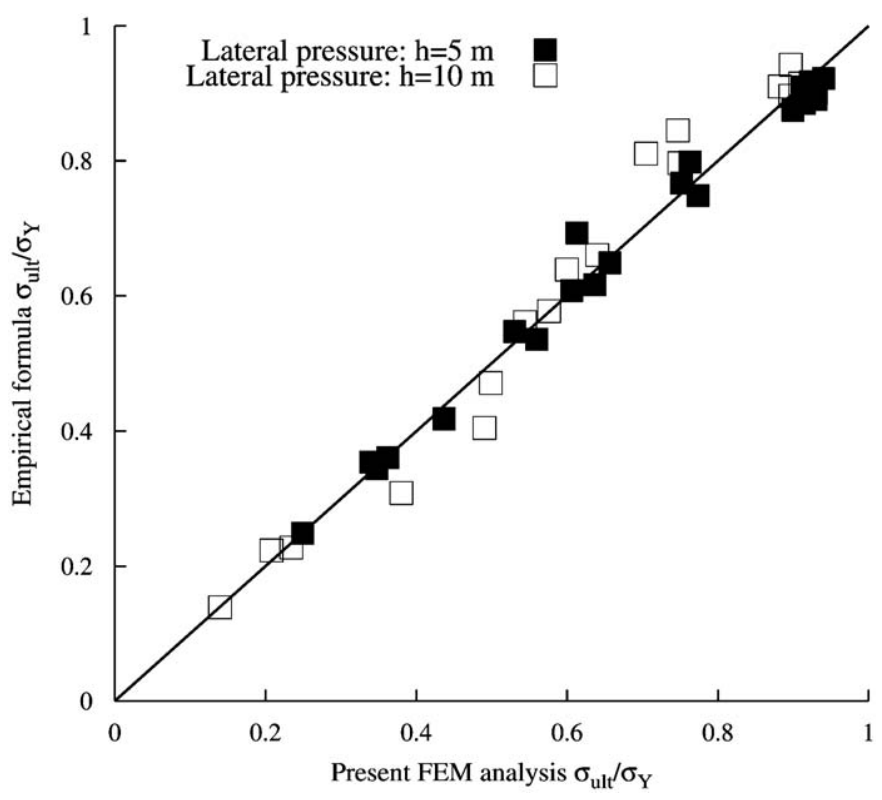

Fig. 9. Comparison of the non-dimensionalised ultimate strength values for aluminium plates stiffened with T-bar stiffeners (as indicated in Tables 2 and 3) under combined in-plane axial compression and lateral pressure.

For the case of aluminium plate with flat-bar stiffeners under water head $=h=0 \mathrm{~m}$

$\frac{\sigma_{u l t}}{\sigma_{\text {Yseq }}}=\frac{1}{\sqrt{1.3551+0.1107 \beta^{2}+0.0814 \lambda^{2}+0.3423 \beta^{2} \lambda^{2}-0.2031 \lambda^{4}}} \leq \frac{1}{\lambda^{2}}$

For the case of aluminium plate with flat-bar stiffeners under water head $=h=5 \mathrm{~m}$

$\frac{\sigma_{u l t}}{\sigma_{\text {Yseq }}}=\frac{1}{\sqrt{1.2719+0.1726 \beta^{2}-0.227 \lambda^{2}+0.3854 \beta^{2} \lambda^{2}+0.0804 \lambda^{4}}} \leq \frac{1}{\lambda^{2}}$

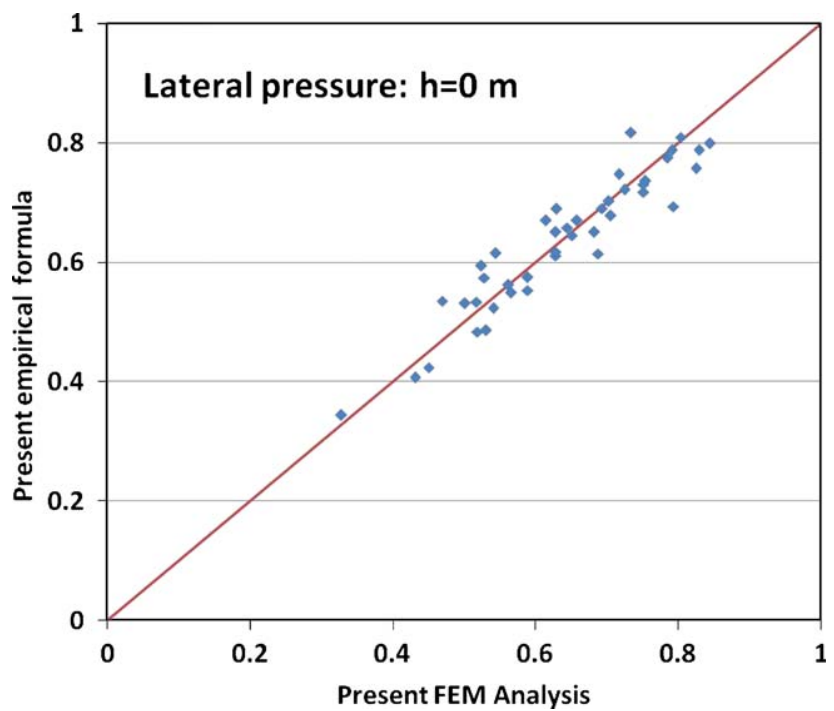

Fig. 10. Comparison of the non-dimensionalised ultimate strength values for aluminium plates stiffened with flat-bar stiffeners (as indicated in Table 4) under pure in-plane axial compression.

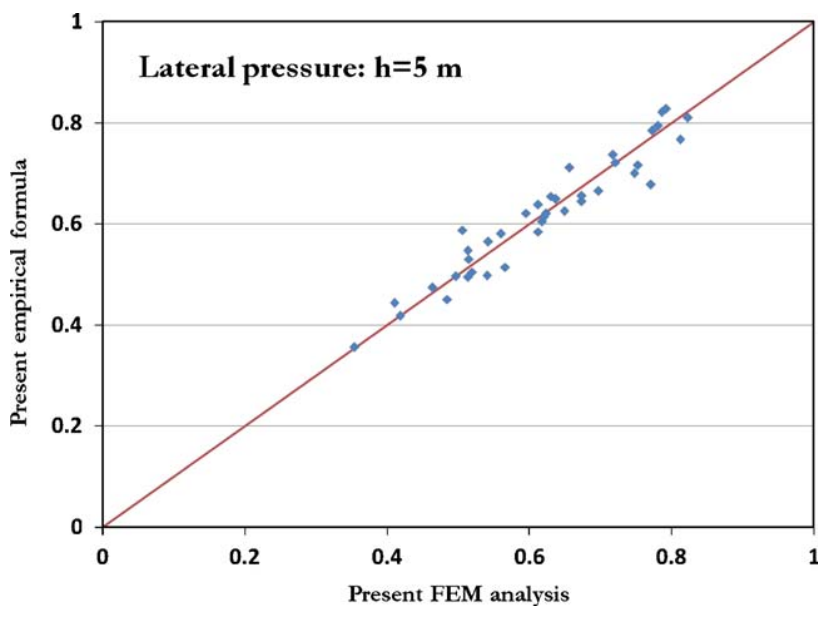

Fig. 11. Comparison of the non-dimensionalised ultimate strength values for aluminium plates stiffened with flat-bar stiffeners (as indicated in Table 5) under combined in-plane axial compression and lateral pressure.

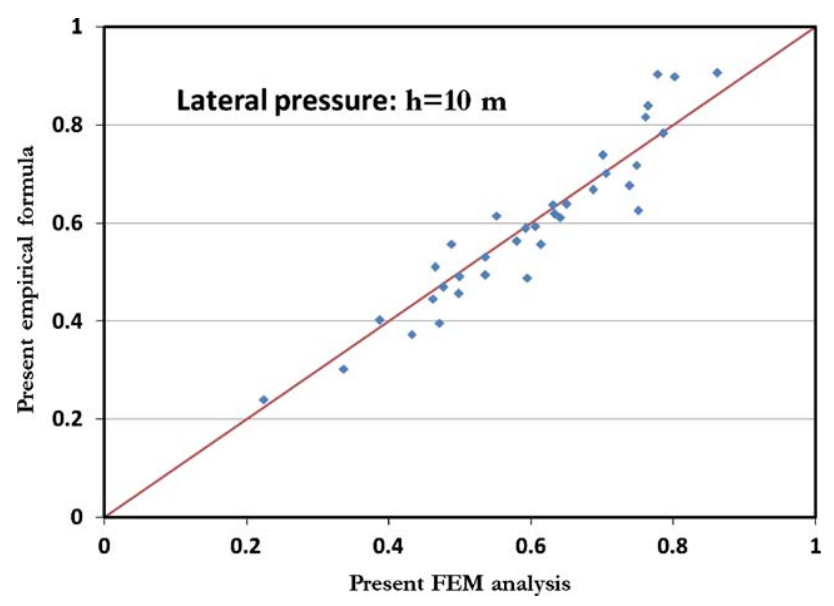

Fig. 12. Comparison of the non-dimensionalised ultimate strength values for aluminium plates stiffened with flat-bar stiffeners (as indicated in Table 6) under combined in-plane axial compression and lateral pressure. 
For the case of aluminium plate with flat-bar stiffeners under water head $=h=10 \mathrm{~m}$

$\frac{\sigma_{u l t}}{\sigma_{\text {Yseq }}}=\frac{1}{\sqrt{1.0421+0.1787 \beta^{2}-1.1282 \lambda^{2}+1.2343 \beta^{2} \lambda^{2}+0.0409 \lambda^{4}}} \leq \frac{1}{\lambda^{2}}$

Accuracy of Eqs. (14)-(16) has been shown, respectively, in Figs. 10-12. A remarkable agreement is observed between two sets of ultimate strength values: calculated by FEM and predicted using the empirical equations.

\subsection{Summarised ultimate strength formulations}

For the convenience of designers and researchers who use the proposed empirical formulations, the coefficients are described as functions of lateral pressure $h$. The resulting formulations are as follows:

$\frac{\sigma_{u l t}}{\sigma_{\text {Yseq }}}=\frac{1}{\sqrt{c_{1}+c_{2} \beta^{2}+c_{3} \lambda^{2}+c_{4} \lambda^{2} \beta^{2}+c_{5} \lambda^{4}}} \leq \frac{1}{\lambda^{2}}$ where for the case of aluminium plate with T-bar stiffeners

$$
\left\{\begin{array}{l}
c_{1}=-0.001 h^{2}+0.071 h+0.731 \\
c_{2}=-0.023 h+0.162 \\
c_{3}=-0.057 h^{2}+0.235 h+0.483 \\
c_{4}=0.004 h^{2}+0.016 h+0.014 \\
c_{5}=0.033 h^{2}-0.151 h+0.096
\end{array}\right.
$$

where for the case of aluminium plate with flat-bar stiffeners

$$
\left\{\begin{array}{l}
c_{1}=-0.002 h^{2}-0.002 h+1.355 \\
c_{2}=-0.001 h^{2}+0.018 h+0.110 \\
c_{3}=0.008 h^{2}-0.102 h+0.081 \\
c_{4}=0.016 h^{2}-0.072 h+0.342 \\
c_{5}=-0.006 h^{2}+0.089 h-0.203
\end{array}\right.
$$

Applying Eqs. (17)-(19), it is possible to estimate the ultimate compressive strength of stiffened aluminium plates under any value of water head $h$ in meters.

Table 7

Comparison of the ultimate strength predictions for continuous stiffened aluminium plates using developed empirical formulations and other formulations (stiffener type:

\begin{tabular}{|c|c|c|c|c|c|c|c|c|}
\hline Stiffener type & ID & $\beta$ & $\lambda$ & $\left(\frac{\sigma_{u l t}}{\sigma_{Y s e q}}\right)_{F E M}$ & $\left(\frac{\sigma_{u l t}}{\sigma_{\text {Yseq }}}\right)_{\text {present formula }}$ & $\left(\frac{\sigma_{\text {ult }}}{\sigma_{\text {Yseq }}}\right)_{D N V}$ & $\left(\frac{\sigma_{u l t}}{\sigma_{Y s e q}}\right)_{\text {Paik and Duran }}$ & $\left(\frac{\sigma_{\text {ult }}}{\sigma_{\text {Yseq }}}\right)_{\text {Paik }}$ \\
\hline 1 & 2 & 3 & 4 & 5 & 6 & 7 & 8 & 9 \\
\hline $\mathrm{T}$ & 1 & 2.4296 & 1.3183 & 0.6239 & 0.5801 & 0.5900 & 0.3488 & 0.3447 \\
\hline $\mathrm{T}$ & 2 & 2.4296 & 0.8773 & 0.6958 & 0.6764 & 0.6132 & 0.5452 & 0.4809 \\
\hline $\mathrm{T}$ & 3 & 2.4296 & 0.6587 & 0.7197 & 0.7152 & 0.6192 & 0.6449 & 0.5470 \\
\hline $\mathrm{T}$ & 4 & 2.4296 & 0.4308 & 0.8007 & 0.7457 & 0.6529 & 0.7191 & 0.6016 \\
\hline $\mathrm{T}$ & 5 & 2.4296 & 0.3226 & 0.8284 & 0.7560 & 0.6662 & 0.7406 & 0.6203 \\
\hline $\mathrm{T}$ & 6 & 2.4296 & 0.2617 & 0.8554 & 0.7606 & 0.6741 & 0.7491 & 0.6284 \\
\hline $\mathrm{T}$ & 7 & 2.4296 & 0.2234 & 0.8626 & 0.7629 & 0.6990 & 0.7533 & 0.6327 \\
\hline $\mathrm{T}$ & 8 & 1.6197 & 1.4620 & 0.6621 & 0.6071 & 0.8011 & 0.2992 & 0.2976 \\
\hline $\mathrm{T}$ & 9 & 1.6197 & 0.9584 & 0.7681 & 0.7629 & 0.8279 & 0.5163 & 0.4585 \\
\hline $\mathrm{T}$ & 10 & 1.6197 & 0.7152 & 0.8476 & 0.8306 & 0.8488 & 0.6502 & 0.5556 \\
\hline $\mathrm{T}$ & 11 & 1.6197 & 0.4458 & 0.8928 & 0.8891 & 0.8366 & 0.7733 & 0.6595 \\
\hline $\mathrm{T}$ & 12 & 1.6197 & 0.3254 & 0.9112 & 0.9077 & 0.8256 & 0.8088 & 0.6971 \\
\hline $\mathrm{T}$ & 13 & 1.6197 & 0.2591 & 0.9247 & 0.9156 & 0.8115 & 0.8227 & 0.7139 \\
\hline $\mathrm{T}$ & 14 & 1.6197 & 0.2175 & 0.9395 & 0.9197 & 0.7978 & 0.8296 & 0.7228 \\
\hline $\mathrm{T}$ & 15 & 2.2777 & 1.9764 & 0.4004 & 0.4373 & 0.5914 & 0.1814 & 0.2009 \\
\hline $\mathrm{T}$ & 16 & 2.2777 & 1.2918 & 0.5865 & 0.6002 & 0.6052 & 0.3589 & 0.3504 \\
\hline $\mathrm{T}$ & 17 & 2.2777 & 0.9574 & 0.6736 & 0.6792 & 0.6135 & 0.5085 & 0.4561 \\
\hline $\mathrm{T}$ & 18 & 2.2777 & 0.5473 & 0.7843 & 0.7560 & 0.6301 & 0.6956 & 0.5853 \\
\hline $\mathrm{T}$ & 19 & 2.2777 & 0.3758 & 0.7854 & 0.7775 & 0.6408 & 0.7438 & 0.6253 \\
\hline $\mathrm{T}$ & 20 & 2.2777 & 0.2836 & 0.8182 & 0.7859 & 0.6492 & 0.7603 & 0.6412 \\
\hline $\mathrm{T}$ & 21 & 2.2777 & 0.2240 & 0.8495 & 0.7902 & 0.6655 & 0.7679 & 0.6492 \\
\hline $\mathrm{T}$ & 22 & 1.2148 & 1.5502 & 0.6648 & 0.6040 & 0.7675 & 0.2724 & 0.2733 \\
\hline $\mathrm{T}$ & 23 & 1.2148 & 1.0199 & 0.8122 & 0.7904 & 0.8179 & 0.4866 & 0.4354 \\
\hline $\mathrm{T}$ & 24 & 1.2148 & 0.7622 & 0.8250 & 0.8780 & 0.8691 & 0.6340 & 0.5439 \\
\hline $\mathrm{T}$ & 25 & 1.2148 & 0.4625 & 0.8867 & 0.9606 & 0.9270 & 0.7903 & 0.6787 \\
\hline $\mathrm{T}$ & 26 & 1.2148 & 0.3318 & 0.9220 & 0.9863 & 0.9849 & 0.8359 & 0.7297 \\
\hline $\mathrm{T}$ & 27 & 1.2148 & 0.2604 & 0.9310 & 0.9971 & 0.9804 & 0.8537 & 0.7527 \\
\hline $\mathrm{T}$ & 28 & 1.2148 & 0.2153 & 0.9475 & 1.0026 & 0.9760 & 0.8625 & 0.7650 \\
\hline $\mathrm{T}$ & 29 & 1.8222 & 1.9991 & 0.4472 & 0.4501 & 0.7166 & 0.1772 & 0.1938 \\
\hline $\mathrm{T}$ & 30 & 1.8222 & 1.3486 & 0.6335 & 0.6253 & 0.7410 & 0.3379 & 0.3297 \\
\hline $\mathrm{T}$ & 31 & 1.8222 & 1.0124 & 0.7509 & 0.7222 & 0.7623 & 0.4860 & 0.4380 \\
\hline $\mathrm{T}$ & 32 & 1.8222 & 0.5776 & 0.8782 & 0.8280 & 0.7689 & 0.7095 & 0.6010 \\
\hline $\mathrm{T}$ & 33 & 1.8222 & 0.3938 & 0.9039 & 0.8590 & 0.7736 & 0.7755 & 0.6604 \\
\hline $\mathrm{T}$ & 34 & 1.8222 & 0.2943 & 0.9172 & 0.8713 & 0.7741 & 0.7990 & 0.6855 \\
\hline $\mathrm{T}$ & 35 & 1.8222 & 0.2293 & 0.9364 & 0.8775 & 0.7668 & 0.8100 & 0.6986 \\
\hline $\mathrm{T}$ & 36 & 3.6444 & 1.9875 & 0.3776 & 0.3760 & 0.4404 & 0.1822 & 0.2180 \\
\hline $\mathrm{T}$ & 37 & 3.6444 & 1.4133 & 0.4611 & 0.4648 & 0.4421 & 0.3164 & 0.3412 \\
\hline $\mathrm{T}$ & 38 & 3.6444 & 1.1048 & 0.5086 & 0.5090 & 0.4646 & 0.4272 & 0.4164 \\
\hline $\mathrm{T}$ & 39 & 3.6444 & 0.8763 & 0.5378 & 0.5372 & 0.4818 & 0.5149 & 0.4623 \\
\hline $\mathrm{T}$ & 40 & 3.6444 & 0.5201 & 0.6087 & 0.5701 & 0.5167 & 0.6113 & 0.5026 \\
\hline $\mathrm{T}$ & 41 & 3.6444 & 0.4023 & 0.6255 & 0.5775 & 0.5402 & 0.6268 & 0.5085 \\
\hline $\mathrm{T}$ & 42 & 3.0370 & 1.5798 & 0.4797 & 0.4781 & 0.4834 & 0.2666 & 0.2877 \\
\hline $\mathrm{T}$ & 43 & 3.0370 & 0.8734 & 0.5597 & 0.6023 & 0.5054 & 0.5324 & 0.4731 \\
\hline $\mathrm{T}$ & 44 & 3.0370 & 0.5421 & 0.6584 & 0.6431 & 0.5653 & 0.6480 & 0.5390 \\
\hline
\end{tabular}
T-bar, lateral pressure: $H=0 \mathrm{~m}$ ). 
3.6. Comparison of ultimate strength formulations with other existing formulations

A comparison is made among the existing formulations and also developed formulations in order to estimate the ultimate strength of stiffened aluminium plates. The developed formulations (Eqs. (17)-(19)) are capable of estimating the ultimate compressive strength of stiffened aluminium plates considering lateral pressure loads. The Paik formulae [23] (Eqs. (3) and (4)) give the ultimate strength of stiffened aluminium plates under pure in-plane compression. The earlier version of empirical formulation developed by Paik and Duran [35], written below, is also examined for estimation of the ultimate strength of aluminium plates stiffened with T-bar stiffeners under pure inplane compression.

$\frac{\sigma_{u l t}}{\sigma_{\text {Yseq }}}=\frac{1}{\sqrt{1.148+0.096 \beta^{2}+1.180 \lambda^{2}-0.052 \lambda^{2} \beta^{2}+1.651 \lambda^{4}}} \leq \frac{1}{\lambda^{2}}$

Also, reference should be made to the DNV formula for estimation of the ultimate compressive strength of stiffened aluminium plates. According to the DNV rules for classification of high speed and light craft [36], the ultimate strength of stiffened aluminium plates under pure in-plane axial compression is given by

$\sigma_{u l t}=P_{\text {ult }} /\left(A_{P}+A_{S}\right)$

where

$P_{u l t}=0.1 \eta_{P}\left(A_{P}+A_{S}\right) \sigma_{e l}+0.1\left(\eta_{S} \sigma_{c}-\eta_{P} \sigma_{e l}\right)\left(\frac{b_{e}}{b} A_{P}+A_{S}\right)[\mathrm{kN}$

$\frac{b_{e}}{b}=\frac{\sigma_{u}-\sigma_{e l}}{\sigma_{f}-\sigma_{e l}}$

$\sigma_{U}=\sigma_{e l}\left[1+0.375\left(\frac{\sigma_{f}}{\sigma_{e l}}-2\right)\right]$

$\sigma_{e l}=\min \left(\sigma_{c \text {-plate }}, \sigma_{c \text {-lateral }}, \sigma_{c \text {-torsional }}, \sigma_{c \text {-web }}, \sigma_{c \text {-flange }}\right)$

Details for calculation of other parameters that exist in the above formulae are given in the DNV rules for classification of high speed and light craft [36]. Elastic buckling strength of stiffened aluminium plate is to be calculated based on Eq. (25) considering the minimum value among their buckling strengths for different modes of buckling failure.

Table 8

Comparison of the ultimate strength predictions for continuous stiffened aluminium plates using developed empirical formulations and other formulations (stiffener type: F-bar, lateral pressure: $H=0 \mathrm{~m}$ ).

\begin{tabular}{|c|c|c|c|c|c|c|c|}
\hline Stiffener type & ID & $\beta$ & $\lambda$ & $\left(\frac{\sigma_{\text {ult }}}{\sigma_{\text {Yseq }}}\right)_{\text {FEM }}$ & $\left(\frac{\sigma_{u l t}}{\sigma_{Y s e q}}\right)_{\text {present formula }}$ & $\left(\frac{\sigma_{\text {ult }}}{\sigma_{\text {Yseq }}}\right)_{\text {Paik }}$ & $\left(\frac{\sigma_{u l t}}{\sigma_{Y s e q}}\right)_{D N V}$ \\
\hline 1 & 2 & 3 & 4 & 5 & 6 & 7 & 8 \\
\hline $\mathrm{F}$ & 1 & 0.9718 & 1.3734 & 0.7336 & 0.8164 & 0.4109 & 1.3823 \\
\hline $\mathrm{F}$ & 2 & 0.9718 & 0.7144 & 0.8291 & 0.7873 & 0.6167 & 1.3910 \\
\hline $\mathrm{F}$ & 3 & 0.9718 & 0.5547 & 0.8450 & 0.7994 & 0.6282 & 1.4368 \\
\hline F & 4 & 0.9718 & 0.4277 & 0.8036 & 0.8093 & 0.6294 & 1.3829 \\
\hline $\mathrm{F}$ & 5 & 1.8222 & 1.7350 & 0.5014 & 0.5309 & 0.2803 & 0.7162 \\
\hline $\mathrm{F}$ & 6 & 1.8222 & 1.3525 & 0.5892 & 0.5529 & 0.4018 & 0.7263 \\
\hline $\mathrm{F}$ & 7 & 1.8222 & 0.5574 & 0.7929 & 0.6931 & 0.5277 & 0.7431 \\
\hline F & 8 & 1.8222 & 0.4321 & 0.7515 & 0.7174 & 0.3960 & 0.7570 \\
\hline $\mathrm{F}$ & 9 & 1.8222 & 0.3211 & 0.7537 & 0.7360 & 0.3362 & 0.7535 \\
\hline $\mathrm{F}$ & 10 & 2.2777 & 1.6906 & 0.4502 & 0.4234 & 0.2861 & 0.5947 \\
\hline F & 11 & 2.2777 & 0.6507 & 0.6285 & 0.6109 & 0.5720 & 0.6176 \\
\hline $\mathrm{F}$ & 12 & 2.2777 & 0.4902 & 0.6286 & 0.6504 & 0.3508 & 0.6278 \\
\hline $\mathrm{F}$ & 13 & 2.2777 & 0.4075 & 0.6150 & 0.6693 & 0.3041 & 0.6327 \\
\hline $\mathrm{F}$ & 14 & 2.2777 & 0.3064 & 0.6295 & 0.6897 & 0.2663 & 0.6506 \\
\hline $\mathrm{F}$ & 15 & 3.0370 & 0.7827 & 0.5185 & 0.4831 & 0.5156 & 0.5006 \\
\hline $\mathrm{F}$ & 16 & 3.0370 & 0.5995 & 0.5178 & 0.5335 & 0.3584 & 0.5059 \\
\hline $\mathrm{F}$ & 17 & 3.0370 & 0.4554 & 0.5278 & 0.5736 & 0.2588 & 0.5271 \\
\hline $\mathrm{F}$ & 18 & 3.0370 & 0.3798 & 0.5238 & 0.5935 & 0.2314 & 0.5302 \\
\hline F & 19 & 3.0370 & 0.2909 & 0.5442 & 0.6145 & 0.2081 & 0.5705 \\
\hline $\mathrm{F}$ & 20 & 1.2148 & 1.3268 & 0.7248 & 0.7214 & 0.4249 & 1.0551 \\
\hline $\mathrm{F}$ & 21 & 1.2148 & 0.6804 & 0.8249 & 0.7567 & 0.6132 & 1.0618 \\
\hline $\mathrm{F}$ & 22 & 1.2148 & 0.5283 & 0.7851 & 0.7747 & 0.6222 & 1.0860 \\
\hline $\mathrm{F}$ & 23 & 1.2148 & 0.4099 & 0.7912 & 0.7878 & 0.6230 & 1.0575 \\
\hline F & 24 & 1.6197 & 0.4979 & 0.7515 & 0.7301 & 0.5274 & 0.8246 \\
\hline $\mathrm{F}$ & 25 & 1.6197 & 0.3915 & 0.7168 & 0.7473 & 0.4273 & 0.8210 \\
\hline $\mathrm{F}$ & 26 & 3.6444 & 1.1344 & 0.3283 & 0.3441 & 0.4064 & 0.4417 \\
\hline F & 27 & 3.6444 & 0.8497 & 0.4314 & 0.4062 & 0.4721 & 0.4469 \\
\hline $\mathrm{F}$ & 28 & 3.6444 & 0.5573 & 0.5310 & 0.4855 & 0.2772 & 0.4712 \\
\hline $\mathrm{F}$ & 29 & 3.6444 & 0.4275 & 0.5412 & 0.5224 & 0.2171 & 0.4775 \\
\hline $\mathrm{F}$ & 30 & 3.6444 & 0.3271 & 0.5661 & 0.5490 & 0.1904 & 0.5041 \\
\hline F & 31 & 3.6444 & 0.2736 & 0.5624 & 0.5616 & 0.1797 & 0.5078 \\
\hline F & 32 & 3.6444 & 0.2123 & 0.5890 & 0.5742 & 0.1694 & 0.5568 \\
\hline F & 33 & 2.6031 & 0.4697 & 0.6279 & 0.6172 & 0.2999 & 0.5566 \\
\hline $\mathrm{F}$ & 34 & 2.6031 & 0.3550 & 0.6510 & 0.6449 & 0.2525 & 0.5721 \\
\hline F & 35 & 2.6031 & 0.2955 & 0.6448 & 0.6575 & 0.2353 & 0.5755 \\
\hline F & 36 & 2.6031 & 0.2240 & 0.6583 & 0.6704 & 0.2187 & 0.6049 \\
\hline $\mathrm{F}$ & 37 & 2.2777 & 0.9677 & 0.4691 & 0.5352 & 0.5138 & 0.6003 \\
\hline $\mathrm{F}$ & 38 & 2.2777 & 0.6369 & 0.6877 & 0.6144 & 0.5452 & 0.6106 \\
\hline $\mathrm{F}$ & 39 & 2.2777 & 0.4880 & 0.6825 & 0.6509 & 0.3492 & 0.6176 \\
\hline $\mathrm{F}$ & 40 & 2.2777 & 0.3677 & 0.7053 & 0.6778 & 0.2874 & 0.6278 \\
\hline $\mathrm{F}$ & 41 & 2.2777 & 0.3056 & 0.6935 & 0.6899 & 0.2661 & 0.6327 \\
\hline F & 42 & 2.2777 & 0.2298 & 0.7030 & 0.7024 & 0.2455 & 0.6506 \\
\hline
\end{tabular}


a

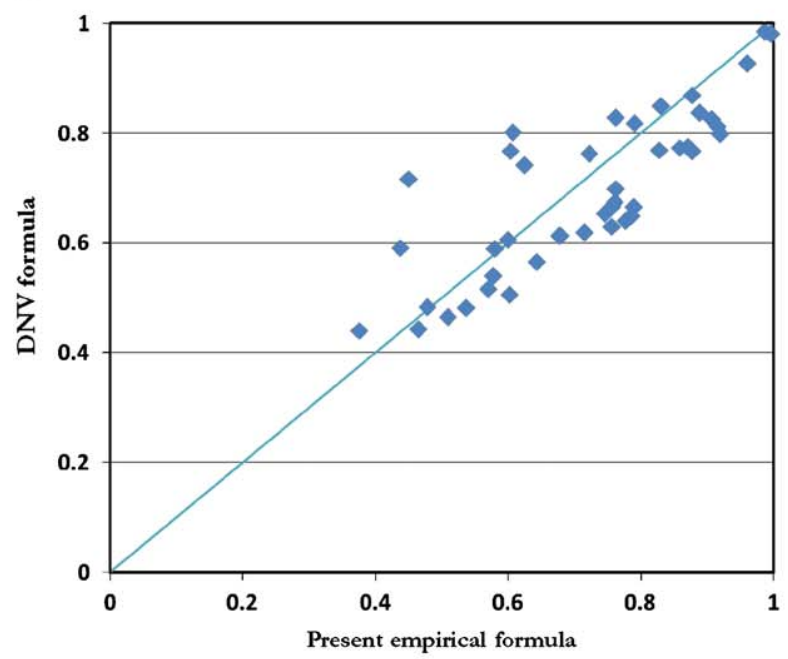

b

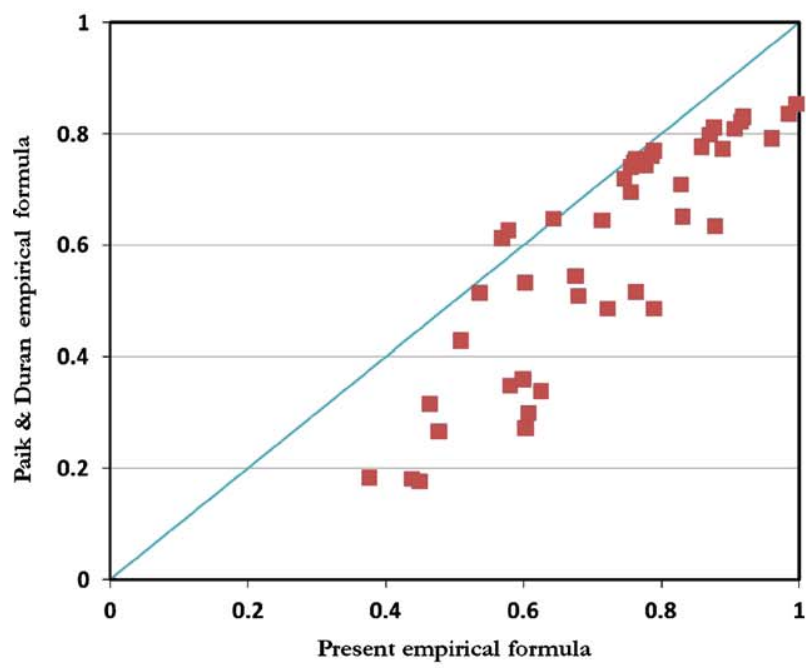

C

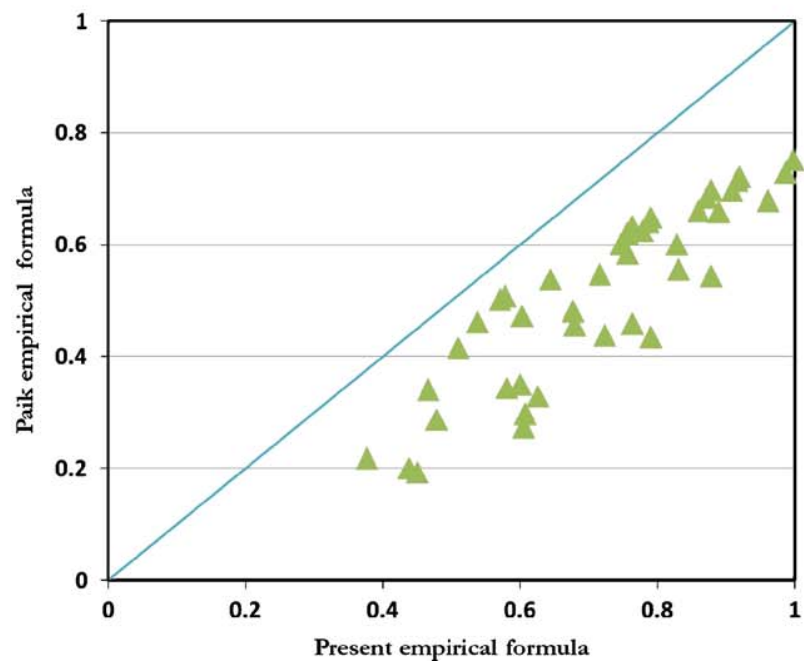

Fig. 13. Comparison of the ultimate strength predictions for aluminium plates stiffened with T-bar stiffeners (as indicated in Table 7) under pure in-plane axial compression.

A comparison of the ultimate strength predictions using developed formulations and also other existing formulations for the two cases of aluminium plates stiffened with T-bar and a

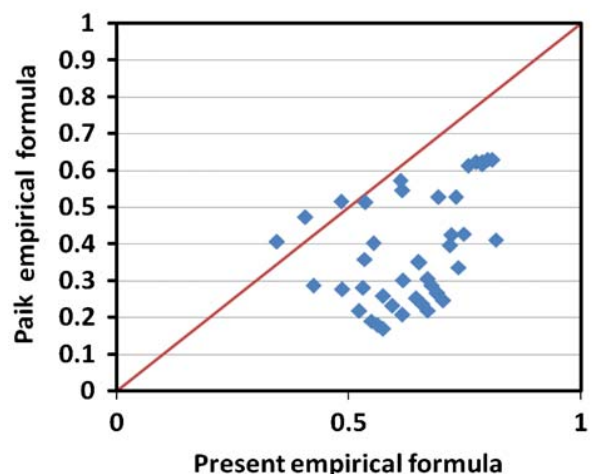

b

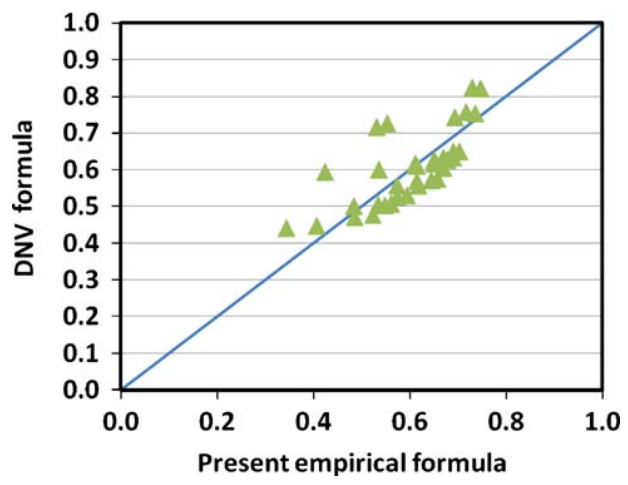

Fig. 14. Comparison of the ultimate strength predictions for aluminium plates stiffened with flat-bar stiffeners (as indicated in Table 8) under pure in-plane axial compression.

flat-bar stiffeners is given, respectively, in Tables 7 and 8. Also, Figs. 13 and 14 give a check of the accuracy of available formulae in comparison with each other. As can be seen, predictions of the present formulations are in good agreement with those made by the DNV formula. On the other hand, predictions made using Paik formulation [23] have some deviations with reference to those made using the authors' formulations. The earlier version of Paik and Duran formulation [35] gives results more or less the same as those of the formulations developed by the authors. All the comparisons presented in Tables 7 and 8 and also in Figs. 13 and 14 are just for the case of pure in-plane compression.

The derived formulations are found to be very effective also in terms of time. Estimation of ultimate strength of continuous stiffened aluminium plates using presented formulations takes a few milliseconds. Besides, modelling of a continuous stiffened aluminium plate with all details, using any commercial finite element code like ANSYS, for an expert and skilled user takes about two or three working days, while the CPU time for its analysis for capturing the value of ultimate strength, including post-processing jobs, is about $30-40 \mathrm{~min}$.

\section{Conclusions}

The aim of the present paper has been to develop closed-form formulations for predicting ultimate compressive strength of stiffened aluminium plates under combined in-plane compression and lateral pressure. Extensive numerical results on welded stiffened aluminium plate structures obtained through a series of elastic-plastic large deflection FEM analyses were used for the present purpose. 
Different forms of ultimate strength expressions were derived for plates with flat-bar or angle/T-bar stiffeners. The ultimate strength formulations developed implicitly take into account effects of weld induced initial imperfections.

The accuracy of derived formulations was checked by a comparison with numerical results. The empirical formulations derived in the present study will be useful for ultimate-strengthbased reliability analyses of aluminium high-speed ship structures. In using the derived empirical formulations for final sizing or detailed strength check calculations, precautions are required such as additional safety factors given the potential for nonconservative strength predictions.

\section{Appendix. Regression method}

\section{A.1. Simple linear regression}

A simple linear regression model for describing the relation between an independent input variable $x$ and output function $y$ can written as

$y=\beta_{1} x+\beta_{0}$

Let us assume there are $n$ independent variables $x_{i}$, where $i=1,2, \ldots, n$. Their corresponding functional values are $y_{i}$. Also, maximum and minimum values of $x_{i}$ are, respectively, $x_{\text {max }}$ and $x_{\text {min. }}$. Estimated values for $\beta_{0}$ and $\beta_{0}$ are, respectively, assumed to be $\beta$ and $\beta$, where they can be found from the following equations:

$\widehat{y}=\widehat{y}(x)=\widehat{\beta} x+\underset{0}{\beta}, x_{\min } \leq x \leq x_{\max }$

$\hat{\beta}=S_{x y} / S_{x x}$

$\widehat{\beta}=\overline{0}-\widehat{\beta} \bar{x}$

where

$\bar{x}=\frac{1}{n} \sum_{i=1}^{n} x_{i}$

$\bar{y}=\frac{1}{n} \sum_{i=1}^{n} y_{i}$

$S_{x x}=\sum_{i=1}^{n} x_{i}^{2}-n \bar{x}^{2}$

$S_{x y}=\sum_{i=1}^{n} x_{i} y_{i}-n \bar{x} \bar{y}$

One way to control the accuracy of the model is to check the distribution of the residuals $e_{i}$, where their values are calculated from the following equation:

$e_{i}=y_{i}-\widehat{y}\left(x_{i}\right), i=1,2, \ldots, n$

If residuals $e_{i}$ have a normal distribution, then it can be concluded that the adopted regression model has considerable accuracy. Another way to check the model is to calculate the quantity $R^{2}$ as follows:

$R^{2}=1-\frac{S S_{E}}{S_{y y}}$ where

$S S_{E}=S_{y y}-\widehat{\beta} S_{x y}$

$S_{y y}=\sum_{i=1}^{n} y_{i}^{2}-n \bar{y}^{2}$

If the value of this quantity in percent, i.e. $100 R^{2}$, is near $100 \%$, it will be a proof of the good regression model.

\section{A.2. Multiple linear regression}

Now, assume a process with a single output $y$ and $k$ input variables $x_{j}$, where $j=1,2, \ldots, k$. This process may be modelled using regression analysis with the following model:

$y=\beta_{0}+\sum_{j=1}^{k} \beta_{j} x_{j}$

Let us assume there are $n$ experimental data, where $n>k$. The independent variables in such a case are represented by $x_{i j}$, where $i=1,2, \ldots, n$ and $j=1,2, \ldots, k$. Their corresponding functional values are $y_{i}$ calculated as follows:

$y_{i}=\beta_{0}+\sum_{j=1}^{k} \beta_{j} x_{i j}, i=1,2, \ldots, n$

If $\widehat{\beta}$ and $\hat{y}$ represent, respectively, the estimated values for $\beta_{i}$ and $y_{i}^{j}$, then the following equation can be written:

$\widehat{y}=\underset{i}{\hat{\beta}}+\sum_{j=1}^{k} \widehat{\beta} x_{i j}, i=1,2, \ldots, n$

or in matrix notation

$Y=X \widehat{\beta}$

where

$X=\left[\begin{array}{ccccc}1 & x_{11} & x_{12} & \cdots & x_{1 k} \\ 1 & x_{21} & x_{22} & \cdots & x_{2 k} \\ \vdots & \vdots & \vdots & \vdots & \vdots \\ 1 & x_{n 1} & x_{n 2} & \cdots & x_{n k}\end{array}\right], Y=\left[\begin{array}{c}y_{1} \\ y_{2} \\ \vdots \\ y_{n}\end{array}\right], \widehat{\beta}=\left[\begin{array}{c}\hat{\beta} \\ 0 \\ \hat{\beta} \\ 1 \\ \vdots \\ \hat{\beta} \\ k\end{array}\right]$

The vector of estimated values for the constants in Eq. (A.16) is determined using the following equation:

$\widehat{\beta}=\left(X^{T} X\right)^{-1} X^{T} Y$

Both of the afore-mentioned methods are applied to control the accuracy of the multiple linear regression model Eq. (A.13). For the second method, the value of quantity $R^{2}$ is determined based on the following equation:

$R^{2}=1-Y^{T} Y-\frac{\widehat{\beta}^{T} X^{T} Y}{Y^{T} Y-n \bar{y}^{2}}$

\section{References}

[1] Murray NW. Buckling of stiffened panels loaded axially and in bending. Struct Eng 1973;51(8):285-301.

[2] Dorman AP, Dwight JB. Tests on stiffened compression panels and plate panels. In: Proceedings of the international conference on steel box girder bridges, February 1973. London: The Institution of Civil Engineers; 1973. p. 63-75.

[3] Smith CS. Compressive strength of welded steel ship grillages. Trans R Inst Nav Archit 1975;117:325-47.

[4] Dow RS. Testing and analysis of a 1/3-scale welded steel frigate model. In: Smith CS, Dow RS, editors. Advances in marine structures-2. Elsevier; 1991. 
[5] Ghavami K. Experimental study of stiffened plates in compression up to collapse. J Constr Steel Res 1994;28(2):197-222 [Special Brazilian Issue, Guest Editor Khosrow Ghavami].

[6] Sherbourne AN, Liaw CY, Marsh C. Stiffened plates in uniaxial compression, vol. 31. IABSE, 1971. p. 145

[7] Moolani FM, Dowling PJ. Ultimate load behaviour of stiffened plates in compression. Steel plates structures. In: Dowling PJ, Harding JE, Frieze PA, editors. Proceedings of the international conference on steel plated structures, Imperial College. London: Crosby Lockwood Staples; 1976. p. 51-88.

[8] Guedes Soares C, Soreide TH. Behaviour and design of stiffened plates under predominantly compressive loads. Int Shipbuild Prog 1983:341.

[9] Bonello MA, Chryssanthopoulos MK, Dowling PJ. Ultimate strength design of stiffened plates under axial compression and bending. Mar Struct 1993;6:533-52.

[10] Chen Q, Zimmerman TJE, DeGeer D, Kennedy BW. Strength and stability testing of stiffened plate components. Ship Structural Committee Report SSC399, 1997.

[11] Clarke JD, Narayanan R. Buckling of aluminium alloy stiffened plate ship structure. In: Aluminium structures-advances design and construction, Proceedings of the international conference on steel and aluminium structures, Cardiff, July 1987. Elsevier; 1987. p. 81-92.

[12] Aalberg A, Langseth M, Larsen PK. Stiffened aluminium panels subjected to axial compression. Thin Wall Struct 2001;39:861-85.

[13] Aalberg A, Langseth M, Malo KA. Ultimate strength of stiffened aluminium plates. Department of Structural Engineering, Norwegian University of Science and Technology; 1998.

[14] Kristensen QHH, Moan T. Ultimate strength of aluminium plates under biaxial loading. In: Proceedings of the fifth international conference on fast sea transportation, New York, 1999.

[15] Zha Y, Moan T, Hanken E. Experimental and numerical study of torsional buckling of stiffeners in aluminium panels. In: Proceedings of the fifth international conference on ISOPE, Seattle, 2000. p. 249-55.

[16] Zha Y, Moan T, Hanken E. Ultimate strength of stiffened aluminium panels with predominantly torsional failure modes. Thin-Walled Struct 2001;39:631-48.

[17] Zha Y, Moan T. Experimental and numerical prediction of collapse of flatbar stiffeners in aluminium panels. J Struct Eng 2003;129(2):160-8.

[18] Hopperstad OS, Langseth M, Hanssen L. Ultimate compressive strength of plate elements in aluminium: correlation of finite element analyses and tests. Thin-Walled Struct 1998;29:31-46.

[19] Rigo P, et al. Sensitivity analysis on ultimate strength of aluminium stiffened panels. Mar Struct 2003;16:437-68.
[20] Paik JK, et al. Ultimate limit state design technology for aluminum multi-hull ship structures. Trans SNAME 2005;113:270-305.

[21] Paik JK, et al. The statistics of weld induced initial imperfections in aluminum stiffened plate structures for marine applications. Int J Marit Eng 2006;148(4):19-63.

[22] Collette MD. The impact of fusion welds on the ultimate strength of aluminum structures. In: Proceedings of PRADS 2007, Houston, USA.

[23] Paik JK. Empirical formulations for predicting the ultimate compressive strength of welded aluminum stiffened panels. Thin-Walled Struct 2007; 45:171-84.

[24] Sielski RA. Research needs in aluminum structure. Ships Offshore Struct 2008;3(1):57-65.

[25] Paik JK, et al. Mechanical collapse testing on aluminum stiffened plate structures for marine applications. Mar Technol 2008;45(4):228-40.

[26] Paik JK, et al. Mechanical collapse testing on aluminum stiffened panels for marine applications. Ship Structure Committee report, SSC-451 2008, Washington, DC, 〈http://www.shipstructure.org $\rangle$.

[27] Paik JK. Buckling collapse testing of friction stir welded aluminum stiffened plate structures. Ship Structure Committee report SR-1454 2009, Washington, DC, 〈http://www.shipstructure.org $\rangle$.

[28] Khedmati MR, Zareei MR, Rigo P. Sensitivity analysis on the elastic buckling and ultimate strength of continuous stiffened aluminium plates under combined in-plane compression and lateral pressure. Thin-Walled Struct 2009. doi:10.1016/j.tws.2009.04.010.

[29] ANSYS user's manual (Version 7.1). Houston, Swanson Analysis Systems Inc., 2003.

[30] Yao T, Fujikubo M, Yanagihara D, Irisawa M. Consideration on FEM modelling for buckling/plastic collapse analysis of stiffened plates. Trans West-Japan Soc Nav Archit 1998;85:121-8 [in Japanese].

[31] Fujikubo M, Yao T, Varghese B, Zha Y, Yamamura K. Elastic local buckling strength of stiffened plates considering plate/stiffener interaction and lateral pressure. In: Proceedings of the eighth international offshore and polar engineering conference, vol. IV, 1998. p. 292-9.

[32] Khedmati MR. Ultimate strength of ship structural members and systems considering local pressure loads. Dr.Eng. thesis, Graduate School of Engineering, Hiroshima University, 2000.

[33] Varghese B. Buckling/plastic collapse strength of plates and stiffened plates under combined loads. Dr.Eng. thesis, Hiroshima University, 1998.

[34] The MathWorks Inc. MATLAB user's guide. South Natick, MA: The MathWorks, Inc.; 2008

[35] Paik JK, Duran A. Ultimate strength of aluminum plates and stiffened panels for marine applications. Mar Technol 2004;41(3):108-21.

[36] DNV. Rules for classification of high speed and light craft, hull structure design, aluminum alloy. Norway: Det Norske Veritas; 2008. 